\title{
Renormalization of twisted Ramond fields in D1-D5 $\mathrm{SCFT}_{2}$
}

\author{
A.A. Lima, ${ }^{a}$ G.M. Sotkov ${ }^{a}$ and M. Stanishkov ${ }^{b}$ \\ ${ }^{a}$ Department of Physics, Federal University of Espírito Santo, \\ 29075-900, Vitória, Brazil \\ ${ }^{b}$ Institute for Nuclear Research and Nuclear Energy, Bulgarian Academy of Sciences, \\ 1784 Sofia, Bulgaria \\ E-mail: andrealves.fis@gmail.com, gsotkov@gmail.com, \\ marian@inrne.bas.bg
}

ABSTRACT: We explore the $n$-twisted Ramond sector of the deformed two-dimensional $\mathcal{N}=(4,4)$ superconformal $\left(T^{4}\right)^{N} / S_{N}$ orbifold theory, describing bound states of D1-D5 brane system in type IIB superstring. We derive the large- $N$ limit of the four-point function of two R-charged twisted Ramond fields and two marginal deformation operators at the free orbifold point. Specific short-distance limits of this function provide several structure constants, the OPE fusion rules and the conformal dimensions of a few non-BPS operators. The second order correction (in the deformation parameter) to the two-point function of the Ramond fields, defined as double integrals over this four-point function, turns out to be UV-divergent, requiring an appropriate renormalization of the fields. We calculate the corrections to the conformal dimensions of the twisted Ramond ground states at the large- $N$ limit. The same integral yields the first-order deviation from zero of the structure constant of the three-point function of two Ramond fields and one deformation operator. Similar results concerning the correction to the two-point function of bare twist operators and their renormalization are also obtained.

Keywords: Conformal Field Theory, Renormalization Regularization and Renormalons, Conformal Field Models in String Theory

ArXiv EPrint: 2010.00172 


\section{Contents}

1 Introduction 1

2 D1-D5 $\mathrm{SCFT}_{2}$ and $\left(T^{4}\right)^{N} / S_{N}$ orbifold 4

3 Away from the free orbifold $\quad 7$

4 Four-point functions $\quad 9$

4.1 The covering surface 9

$\begin{array}{lll}4.2 & \text { Four-point functions } & 10\end{array}$

4.2.1 Charged Ramond fields 13

$\begin{array}{lll}4.2 .2 & \text { Bare twists } & 15\end{array}$

5 OPE limits, fusion rules and structure constants $\quad \mathbf{1 5}$

5.1 The OPE of two interaction operators 16

5.2 Non-BPS operators in the OPEs of $R_{n}^{ \pm}$with $O_{2}^{(\text {int })} \quad 19$

6 Analytic regularization and field renormalization 21

6.1 Dotsenko-Fateev integrals 21

6.2 The integral for R-charged Ramond fields 25

6.3 The integral for bare twists 28

6.4 Renormalization of Ramond and twist fields 28

6.5 On spectral flow 32

7 Discussion $\quad 34$

A Conventions for the $\mathcal{N}=(4,4)$ SCFT 36

$\begin{array}{ll}\text { B Asymptotics to OPEs } & 37\end{array}$

C OPEs with bare twists and structure constants 38

\section{Introduction}

In the near-horizon decoupling limit [1] of the type-IIB supergravity description of bound states of $N_{1}$ D1- and $N_{5}$ D5-branes, the asymptotic geometry becomes $A d S_{3} \times S^{3} \times T^{4}$, with large Ramond-Ramond charges [2,3], from which one can reconstruct its holographic dual $\mathrm{SCFT}_{2}$; see [4-6] for reviews. There is strong indication that this D1-D5 $\mathrm{SCFT}_{2}$ flows in the infrared to a free field theory whose sigma model is $\left(T^{4}\right)^{N} / S_{N}$, an orbifold of $T^{4}$ by the symmetric group $S_{N}$, with $N=N_{1} N_{5}$, while the supergravity description is obtained by moving in moduli space with a deformation away from this 'free orbifold point'. Gravitational solutions, which include the Strominger-Vafa black hole [7] and fuzzball geometries $[5,6,8-11]$, are all dual to states in the Ramond sector of the $\mathrm{SCFT}_{2}$, and one 
can find correspondences between the geometries and Ramond states in many cases where the latter are BPS-protected from renormalization. Extensive research has achieved considerable progress in the understanding of the free orbifold and its deformation, as well as in the construction of 'superstratum' geometries corresponding to the microscopic picture [12-38]. Nevertheless, the description of the dynamics of the deformed $\mathrm{SCFT}_{2}$ is still not fully understood. One of the open problems concerns the selection rules separating protected states from "lifted" ones, whose conformal data flow in the deformed theory after renormalization [39-44].

The present paper investigates the effects on the conformal properties of twisted ground states in the $\mathcal{N}=(4,4)$ orbifold $\mathrm{SCFT}_{2}$ when the theory is deformed by a marginal scalar modulus operator $\lambda O_{[2]}^{\text {(int) }}[20,21,45-47]$. The first-order correction, in powers of $\lambda$, of the two-point function of a ground state is known to vanish. Our main result is an explicit derivation of the finite part of the second-order correction to two-point functions of $n$ twisted primary operators $\mathscr{O}_{[n]}$, by eliminating the UV divergences with an appropriate renormalization of the fields. As a consequence, the scaling dimension $\Delta_{n}^{\mathscr{O}}(0)$ in the free orbifold point flows with $\lambda$ according to

$$
\Delta_{n}^{\mathscr{O}}(\lambda)=\Delta_{n}^{\mathscr{O}}(0)+\frac{\pi}{2} \lambda^{2}\left|J_{\mathscr{O}}(n)\right|,
$$

where $J_{\mathscr{O}}(n)$ is a regularized integral defined in section 6 below. We will be mostly interested in two specific operators: the bare twist field $\sigma_{[n]}$, and, more importantly, the twisted R-charged Ramond fields $R_{[n]}^{ \pm}$, with bare (holomorphic) conformal weight $h=\frac{n}{4}$. In both cases, our main result - the lifting of the conformal dimensions - holds for $n<N$. Ramond fields with $n=N$ and $n=2$, i.e. with maximal and minimal twist, are protected at leading order in the large- $N$ approximation. Bare twist fields with $n=N$ are also protected at leading order. For the particular case of twisted Ramond fields, this has been recently reported in our short letter [48].

The main ingredient in the calculation of second-order corrections to the two-point functions is finding an explicit analytic expression for the four-point function

$$
\left\langle\mathscr{O}_{[n]}^{\dagger}\left(z_{1}, \bar{z}_{1}\right) O_{[2]}^{(\mathrm{int})}\left(z_{2}, \bar{z}_{2}\right) O_{[2]}^{(\mathrm{int})}\left(z_{3}, \bar{z}_{3}\right) \mathscr{O}_{[n]}\left(z_{4}, \bar{z}_{4}\right)\right\rangle
$$

for the fields $\mathscr{O}_{[n]}$ we are interested in. We will present a detailed derivation of the large- $N$ approximation of the corresponding functions, by applying covering surface techniques [12, 13] combined with the 'stress-tensor method' [49]. Our computation of the leading term in the $1 / N$ expansion of the connected part of (1.2), takes into account only the terms contributing to genus zero surfaces, i.e. we use the well known map $[50,51]$ between the "base" branched sphere to its genus-zero covering surface [12]. The alliance of the covering surface with the stress-tensor method emphasizes some interesting mathematical properties of the correlation functions, and their relation to Hurwitz theory [16, 17, 52].

Corrections to the anomalous dimensions at second-order follow from the integral of (1.2) over the positions of the interaction operators. The analogous integral in the case where there are NS chiral fields at $z_{1}$ and $z_{4}$ has been computed in [17], and shown to vanish, as expected for protected operators which should not renormalize. For the twisted 
Ramond fields and the twist operators, however, the integrand has a more complicated structure, with one more branch cut, and without appropriate regularization the integrals are divergent. In order to define and evaluate their finite parts, we have elaborated a regularization procedure and a specific renormalization scheme for the fields in the deformed $\mathrm{SCFT}_{2}$. Our starting point is the observation that the integrals we are interested in can be put in a form studied by Dotsenko and Fateev [53-55] in a different context, as integral representation of the conformal blocks of primary fields (curiously; not of their integrals) in the $c<1$ series $^{1}$ of minimal $\mathrm{CFT}_{2}$ models. While, in the one hand, they can be formally written as specific contour integrals in the complex plane - with the contours ensuring a series of algebraic properties - on the other hand these integrals can be represented by four 'canonical functions' which are analytic in their parameters, even in cases where the integral itself diverges. Thus, by analytic continuation, the canonical functions give a regularized result for the desired integrals of the four-point functions (1.2). When applied to the parameters of NS chirals, this procedure gives a vanishing result, as expected; but when applied to the Ramond and twisted fields, we find finite, non-vanishing corrections to the conformal dimensions. The analytic expressions for the renormalized conformal dimensions of $R_{[n]}^{ \pm}$and $\sigma_{[n]}$ is one of the most important results of this paper. As a byproduct of the computation of the integrals, we can also present the first-order correction to the structure constants $\left\langle R_{[n]}^{-}(\infty) O_{[2]}^{(\text {int) }}(1) R_{[n]}^{+}(0)\right\rangle$, and $\left\langle\sigma_{[n]}(\infty) O_{[2]}^{(\text {int })}(1) \sigma_{[n]}(0)\right\rangle$, which do not vanish in the deformed $\mathrm{SCFT}_{2}$. It is worthwhile to mention the recent use of similar methods to the renormalization of certain composite Ramond fields, for example $R_{[n]}^{+}(z) R_{[m]}^{-}(z)$ [59]. In the composite case, an important consequence of the renormalization procedure is the existence of a condition, namely $m+n=N$, selecting a class of protected (non-renormalized) states. The remaining states, with $n+m<N$, are lifted: their renormalized conformal dimensions flow with $\lambda$, and are given by the sum of the second-order corrections (1.1) for each one of the constituents, i.e. $\Delta_{n}^{R}(\lambda)+\Delta_{m}^{R}(\lambda)$. What distinguishes the protected Ramond fields from the lifted ones is that the former have conformal weight $h=\frac{1}{24} c_{o r b}=\frac{1}{4} N$, i.e. they are Ramond ground states of the full orbifold theory; in contrast, the fields with $h=\frac{1}{4} n, n<N$ are Ramond ground states only of the $n$-twisted sector, or of the ' $n$-wound component string' in the familiar description of the D1-D5 SCFT in terms of effective stings.

Another contribution of the present paper is the analysis of short-distance limits of the four-point function (1.2). In the limits where operators coincide, $u \rightarrow 0,1, \infty$, we are able to derive several structure constants, the OPE fusion rules and the conformal dimensions of some non-BPS operators. These OPE data add to the description of the Ramond and twisted sectors of the free-orbifold point. Our results for the non-BPS fields are consistent with what is known about the chiral NS and twisted sectors [17, 23]. They are also in agreement with the recently conjectured universality of OPEs of certain chiral fields and the deformation operator in the large- $N$ limit $[60,61]$, and represent an extension of these results for all other sectors of the free orbifold theory. In particular, we find that the OPE algebra of the deformation operator and the Ramond fields includes a set

\footnotetext{
${ }^{1}$ See refs. [56-58] for the extension of the Dotsenko-Fateev integral representation to the Ramond and twisted sectors of the $\mathcal{N}=1$ and $\mathcal{N}=2$ supersymmetric minimal models.
} 
of R-charged twisted non-BPS operators $Y_{m}^{ \pm}$, appearing in the OPEs $O_{[2]}^{(\mathrm{int})}(z, \bar{z}) R_{n}^{ \pm}(0)$. Similarly, the algebra of $O_{[2]}^{(\text {int) }}$ and $\sigma_{n}$ includes new twisted operators $\mathcal{Y}_{m}$. We have calculated the dimensions of these operators, as well as the values of structure constants such as $\left\langle R_{n}^{ \pm}(\infty) O_{2}^{(\mathrm{int})}(1) Y_{n \pm 1}^{ \pm}(0)\right\rangle$. Applying the fractional spectral flows of ref. [61] with $\xi=n /(n+1)$, we find that our results for the twisted Ramond fields' OPEs are in complete correspondence with those obtained from OPEs in the NS sector resulting in specific non-BPS NS fields.

The structure of the paper is as follows. In sections 2 and 3, we fix our notations by defining first the free orbifold $\mathrm{SCFT}_{2}$, and then its deformation away from the free orbifold point; we also review some key features of conformal perturbation theory used later. In section 4, we give a detailed calculation of the four-point functions involving Ramond and bare twist fields, necessary for the second-order correction of the two-point functions. In section 5, we investigate certain short-distance limits of the four-point function in order to extract OPE fusion rules, conformal weights and structure constants of several operators in the free-orbifold point. In section 6 , we return to conformal perturbation theory, with a detailed study of the regularization and the final computation of integrals resulting in the change of the conformal weights of $R_{[n]}^{ \pm}$and $\sigma_{[n]}$; we also explain how the renormalization scheme can be extended to a generic primary field $\mathscr{O}_{[n]}$; we also comment on the spectral flow between the fields $R_{[n]}^{ \pm}$and NS chiral operators in the free theory, and how it is "broken" after the deformation. In section 7 we present a compact summary of our results, together with a short discussion of a few open problems and the eventual consequences of the continuous ( $\lambda$-dependent) conformal dimensions of the renormalized twisted Ramond fields for their geometric bulk counterparts. Some auxiliary topics are left for the appendices.

\section{D1-D5 $\mathrm{SCFT}_{2}$ and $\left(T^{4}\right)^{N} / S_{N}$ orbifold}

The 'free orbifold point' of the D1-D5 system is the $\mathrm{SCFT}_{2}$ with central charge $\left(c_{\text {orb }}, \tilde{c}_{\text {orb }}\right)=$ $(6 N, 6 N)$, obtained by taking $N$ copies of the free $\mathcal{N}=(4,4) \mathrm{SCFT}_{2}$, identified under the symmetric group $S_{N}$, with target space $\left(T^{4}\right)^{N} / S_{N}$. The $\mathcal{N}=(4,4)$ superconformal algebra of the 'seed theory' has central charge $(c, \tilde{c})=(6,6)$, R-symmetry group $\mathrm{SU}(2)_{L} \times \mathrm{SU}(2)_{R}$, and 'internal' group $\mathrm{SO}(4)_{\mathrm{I}}=\mathrm{SU}(2)_{1} \times \mathrm{SU}(2)_{2}$ corresponding to the torus $T^{4}$ of target space. We work on the complex plane, with coordinates $z, \bar{z}$.

The unitary representations of the holomorphic $\mathcal{N}=4$ algebra are characterized by three numbers $\left\{h, j^{3}, \mathfrak{j}^{3}\right\}$, respectively the conformal weight and the semi-integer charges under the R-current $J^{3}(z)$ of $\mathrm{SU}(2)_{L}$, and a current $\mathfrak{J}^{3}(z)$ of the global $\mathrm{SU}(2)_{1}$. Similar numbers $\left\{\tilde{h}, \tilde{j}^{3}, \tilde{j}^{3}\right\}$ characterize the anti-holomorphic sector with $\mathrm{SU}(2)_{R}$ and $\mathrm{SU}(2)_{2}$ groups.

The theory can be realized in terms of free bosons $X^{\dot{A} A}(z, \bar{z})$ and free fermions $\psi^{\alpha \dot{A}}(z)$, $\tilde{\psi}^{\dot{\alpha} \dot{A}}(\bar{z})$, whereas the stress-tensor, the R-current and the super-current are expressed as

$$
\begin{aligned}
T(z) & =\frac{1}{4} \epsilon_{\dot{A} \dot{B}} \epsilon_{A B} \partial X^{\dot{A} A} \partial X^{\dot{B} B}+\frac{1}{4} \epsilon_{\dot{A} \dot{B}} \epsilon_{\alpha \beta} \psi^{\alpha \dot{A}} \partial \psi^{\beta \dot{B}} \\
J^{a}(z) & =\frac{1}{4} \epsilon_{\dot{A} \dot{B}} \epsilon_{\alpha \beta} \psi^{\alpha \dot{A}}\left[\sigma^{* a}\right]_{\gamma}^{\beta} \psi^{\gamma \dot{B}} \\
G^{\alpha A}(z) & =\epsilon_{\dot{A} \dot{B}} \psi^{\alpha \dot{A}} \partial X^{\dot{B} A}
\end{aligned}
$$


with similar expressions for the anti-holomorphic sector. Conventions for SU(2) indices are given in appendix A. The complex bosons and the complex fermions obey reality conditions (A.3), and can be written in terms of real bosons and fermions $X_{i}(z, \bar{z}), \psi_{i}(z)$ and $\tilde{\psi}_{i}(\bar{z}), i=1,2,3,4$; see (A.2). The fermions can be described in terms of chiral scalar bosons $\phi_{r}(z)$ and $\tilde{\phi}_{r}(\bar{z})$, with $r=1,2$. In the holomorphic sector,

$$
\left[\begin{array}{c}
\psi^{+\dot{1}}(z) \\
\psi^{-\dot{1}}(z)
\end{array}\right]=\left[\begin{array}{c}
e^{-i \phi_{2}(z)} \\
e^{-i \phi_{1}(z)}
\end{array}\right], \quad\left[\begin{array}{c}
\psi^{+\dot{2}}(z) \\
\psi^{-\dot{2}}(z)
\end{array}\right]=\left[\begin{array}{c}
e^{i \phi_{1}(z)} \\
-e^{i \phi_{2}(z)}
\end{array}\right] .
$$

Every exponential should be understood to be normal-ordered (and we ignore cocycles). The stress-tensor (2.1a) can be written in the completely bosonic form, ${ }^{2}$

$$
T(z)=-\frac{1}{2} \lim _{z^{\prime} \rightarrow z}\left[\sum_{i=1}^{4} \frac{1}{2} \partial X_{i}(z) \partial X_{i}\left(z^{\prime}\right)+\sum_{r=1}^{2} \partial \phi_{r}(z) \partial \phi_{r}\left(z^{\prime}\right)+\frac{c}{\left(z-z^{\prime}\right)^{2}}\right] .
$$

Bosons are assumed to be periodic, so e.g. $X^{\dot{A} A}\left(e^{2 \pi i} z\right)=X^{\dot{A} A}(z)$. Fermions can have Neveu-Schwarz or Ramond boundary conditions on $\mathbb{C}$. The Ramond sector has a collection of degenerate vacua with holomorphic dimension $h=\frac{c}{24}=\frac{1}{4}$, and different charges under the global and R-symmetry $\mathrm{SU}(2)$ groups. The set of Ramond vacua can be obtained from the NS vacuum by the action of spin fields, conveniently realized as exponentials, e.g. for the $\mathrm{SU}(2)$ doublet $S^{\alpha}(z)$,

$$
S^{ \pm}(z)=e^{ \pm \frac{i}{2}\left[\phi_{1}(z)-\phi_{2}(z)\right]}
$$

To construct the orbifold $\left(T^{4}\right)^{N} / S_{N}$, one makes $N$ copies of the free SCFT and identifies them under the action of $S_{N}$; more explicitly, we take the $N$-fold tensor product $\left(\otimes^{N} T^{4}\right) / S_{N}$, and label operators $\mathscr{O}_{I}$ in each copy by an index $I=1, \cdots, N$. The energy tensor becomes

$$
T(z)=-\frac{1}{2} \lim _{z^{\prime} \rightarrow z} \sum_{I=1}^{N}\left[\sum_{i=1}^{4} \frac{1}{2} \partial X_{i I}(z) \partial X_{i I}\left(z^{\prime}\right)+\sum_{r=1}^{2} \partial \phi_{r I}(z) \partial \phi_{r I}\left(z^{\prime}\right)+\frac{c}{\left(z-z^{\prime}\right)^{2}}\right] .
$$

and the total central charge is $c_{\text {orb }}=N c=6 N$.

Permutations of the copies can be realized by the insertion of twist operators $\sigma_{g}(z)$, $g \in S_{N}$, which give a representation of $S_{N}$, and act on the other operators by twisting their boundary conditions [62],

$$
\mathscr{O}_{I}\left(e^{2 \pi i} z\right) \sigma_{g}(0)=\mathscr{O}_{g(I)}(z) \sigma_{g}(0)
$$

We are going to consider only cyclic twists, which form the building blocks of the Hilbert space of the orbifold theory [63]. So, denoting by $(n)$ a generic cycle of length $n$, we consider $g=(1)^{N-n}(n) \cong(n)$, leaving the trivial cycles implicit. We denote by $\sigma_{n}$ the

\footnotetext{
${ }^{2}$ Normal ordering of two operators $\mathscr{A}_{1}$ and $\mathscr{A}_{2}$ is defined by

$$
: \mathscr{A}_{1}(z) \mathscr{A}_{2}(z): \equiv \lim _{\varepsilon \rightarrow 0}\left[\mathscr{A}_{1}(z+\varepsilon) \mathscr{A}_{2}(z)-\left\langle\mathscr{A}_{1}(z+\varepsilon) \mathscr{A}_{2}(z)\right\rangle\right] .
$$
}


twist operator for a generic cycle of length $n$; they cyclically permute the $n$ copies of the fields appearing in the cycle $(n)$, while leaving the remaining copies invariant. The conjugacy class is represented by the orbit-invariant combination

$$
\sigma_{[n]} \equiv \frac{1}{\mathscr{S}_{n}(N)} \sum_{h \in S_{N}} \sigma_{h^{-1}(n) h}
$$

where the representing cycle can be taken to be $(n)=(1 \cdots n)$, and the combinatorial factor $\mathscr{S}_{n}(N)$ makes the two-point function normalized, i.e.

$$
\left\langle\sigma_{[n]}\left(z_{1}, \bar{z}_{1}\right) \sigma_{[m]}\left(z_{2}, \bar{z}_{2}\right)\right\rangle=\frac{\delta_{m n}}{\left|z_{1}-z_{2}\right|^{2 \Delta_{n}}} .
$$

The well-known (total) conformal dimension of a twist $\sigma_{n}(z, \bar{z})$ is $[13,62]$

$$
\Delta_{n}^{\sigma}=h_{n}^{\sigma}+\tilde{h}_{n}^{\sigma}, \quad h_{n}^{\sigma}=\frac{1}{4}\left(n-\frac{1}{n}\right)=\tilde{h}_{n}^{\sigma} .
$$

The $n$-twisted Ramond sector is generated by twisted spin operators with the appropriate $\frac{1}{n}$ rescaling of their weights. For the representative permutation $(1 \cdots n)$, the $n$-twisted R-charged fields $R_{n}^{\alpha}(z)$ are

$$
R_{n}^{ \pm}(z) \equiv \exp \left( \pm \frac{i}{2 n} \sum_{I=1}^{n}\left[\phi_{1, I}(z)-\phi_{2, I}(z)\right]\right) \sigma_{(1 \cdots n)}(z)
$$

with a similar construction for the neutral $R_{n}^{\dot{A}}(z)$. From these, we can compose $S_{N^{-i n v a r i a n t}}$ combinations $R_{[n]}^{\zeta}(z), \zeta= \pm, \dot{A}$ by summing over orbits, as we did for the normalized $S_{N^{-}}$ invariant twists $\sigma_{[n]}$. For example, the R-charged fields, with which we will be primarily concerned, are written explicitly as

$$
R_{[n]}^{ \pm}(z) \equiv \frac{1}{\mathscr{S}_{n}(N)} \sum_{h \in S_{N}} \exp \left( \pm \frac{i}{2 n} \sum_{I=1}^{n}\left[\phi_{1, h(I)}(z)-\phi_{2, h(I)}(z)\right]\right) \sigma_{h^{-1}(1 \cdots n) h}(z)
$$

where in the exponential we sum over $h(I)=\{h(1), \cdots, h(n)\}$, the image of the original copy set $I=\{1, \cdots, n\}$ under the permutation $h$. The $R_{[n]}^{ \pm}$, like the spin fields $S^{ \pm}$, form a doublet of $\mathrm{SU}(2)_{L}$ and a singlet of $\mathrm{SU}(2)_{1}$, with charges $j^{3}= \pm \frac{1}{2}$ and $\mathfrak{j}^{3}=0$. On the other hand, the $R_{[n]}^{\dot{A}}(z)$ form a singlet of R-symmetry and a doublet of $\mathrm{SU}(2)_{1}$, with charges $j^{3}=0$ and $\mathfrak{j}^{3}= \pm \frac{1}{2}$. The conformal weight of the $R_{n}^{(\zeta)}$ is

$$
h_{n}^{R}=\frac{n}{4},
$$

obtained from the combined weights of the exponential and the twist. Completely analogous fields $\tilde{R}_{[n]}^{\zeta}(\bar{z})$, with dimension $\tilde{h}_{n}^{R}=h_{n}^{R}$, make the anti-holomorphic sector. The normalization factor $\mathscr{S}_{n}(N)$ ensures that the two-point functions are normalized, granted that the non- $S_{N}$-invariant functions are normalized:

$$
\left\langle R_{[n]}^{\mp}(\infty) R_{[n]}^{ \pm}(0)\right\rangle=1=\left\langle R_{n}^{\mp}(\infty) R_{n}^{ \pm}(0)\right\rangle .
$$


Let us examine these fields a little further. The Hilbert space of the orbifold theory, $\mathcal{H}_{\text {orb }}=\oplus_{[g]} \mathcal{H}_{g}$, is a direct sum of sectors invariant under the conjugacy classes of $S_{N}$ [63]. The latter are given by the irreducible decomposition of $g \in S_{N}$ into disjoint cycles, $[g]=$ $\prod_{k \in \mathbb{N}}(k)^{q_{k}}$, with $\sum_{k} k q_{k}=N$, and we are interested in the simplest sector, corresponding to $[g]=(1)^{N-n}(n)$. This Hilbert space, $\mathcal{H}_{(n)}$, is invariant under the centralizer subgroup $S_{N-n} \times \mathbb{Z}_{n}$, where $S_{N-n}$ permutes the $N-n$ trivial cycles (1), and $\mathbb{Z}_{n}$ acts on the elements permuted by $(n)$. It can be further decomposed as [63]

$$
\mathcal{H}_{(n)}=\mathcal{S}^{(N-n)} \otimes \mathcal{H}_{(n)}^{\mathbb{Z}_{n}},
$$

where $\mathcal{S}^{(N-n)}$ is the symmetric tensor product of copies entering the trivial cycles, and where $\mathcal{H}_{(n)}^{\mathbb{Z}_{n}}$ corresponds to the copies permuted by $(n) \in \mathbb{Z}_{n}$. States in $\mathcal{H}_{(n)}$ can be interpreted as a string with winding number $n$. We can think of the construction of the operators $R_{n}^{(\zeta)}$ as exciting the $n$-wound copies to the Ramond ground state, while leaving the unwound copies in the NS vacuum. Thus the operators $R_{n}^{(\zeta)}$ correspond to states $\left|R_{n}^{(\zeta)}\right\rangle=|\varnothing\rangle_{N-1}^{\mathrm{NS}} \otimes|\varnothing, \zeta\rangle_{(n)}^{\mathrm{R}}$ where $|\varnothing\rangle_{N-1}^{\mathrm{NS}} \in \mathcal{S}^{N-1}$ is the NS vacuum of the non-twisted copies and $|\varnothing, \zeta\rangle_{(n)}^{\mathrm{R}}$ are Ramond ground states of a CFT defined on $\mathcal{H}_{(n)}^{\mathbb{Z}_{n}}$. Since the latter CFT involves $n$ copies of the $\mathcal{N}=4$ SCFT, it has central charge $c=6 n$; its Ramond ground states have the conformal weight $\frac{c}{24}=\frac{n}{4}$ in eq. (2.12). We will sometimes refer to $R_{[n]}^{\alpha}$ as 'Ramond ground states', but it should be kept in mind that this is an abuse of nomenclature, as $R_{n}^{\alpha}$ are ground states only of the $n$-wound string; the true Ramond ground states of the orbifold theory have conformal weight $h=\frac{1}{24} c_{o r b}=\frac{1}{4} N$, and are made either by $R_{[N]}^{\alpha}$, i.e. by single-cycle fields with maximal twist $n=N$, or, more generally, by "composite fields" $\prod_{k}\left(R_{n_{k}}^{\alpha_{k}}\right)^{k}$ with $\sum_{k} k n_{k}=N$.

The main objective of this paper is to describe how the dimensions $h_{n}^{R}=\frac{1}{4} n$ are corrected when the free orbifolded SCFT is perturbed by a marginal operator.

\section{Away from the free orbifold}

A marginal deformation of the free orbifold turns the theory into an interacting SCFT, with the action

$$
S_{\text {int }}=S_{\text {free }}+\lambda \int d^{2} z O_{[2]}^{(\text {int })}(z, \bar{z})
$$

parameterized by a dimensionless deformation parameter $\lambda$. In the large- $N$ limit, in which we will be interested, the deformation parameter $\lambda$ should scale with $N$ in such a way that the 't Hooft coupling $\lambda_{*} \equiv \lambda / \sqrt{N}$ is held fixed as $N \rightarrow \infty$; see [12, 52].

The "scalar modulus" interaction operator $O_{[2]}^{(\text {int })}$ is marginal, with total conformal dimension $\Delta=h+\tilde{h}=2$. This dimension should not change under renormalization. Also, $O_{[2]}^{(\text {int })}$ must be a singlet of R-symmetry, in order for $\mathcal{N}=(4,4)$ SUSY not to be broken. From the 20 deformation operators, which correspond to the 20 SUGRA moduli (see [45]), we consider the $S_{N}$-invariant singlet

$$
O_{[2]}^{(\text {int })}(z, \bar{z})=\epsilon_{A B} G_{-\frac{1}{2}}^{-A} \tilde{G}_{-\frac{1}{2}}^{\dot{B}} O_{[2]}^{(0,0)}(z, \bar{z})
$$

constructed as a descendent of the NS chiral field $O_{[2]}^{(0,0)}(z)$ with $h=\frac{1}{2}=j^{3}$. 
Let us review a few key results in conformal perturbation theory used in the next sections; see for example [41] for more detail. For a marginal perturbation, the two-point function

$$
\left\langle\mathscr{O}\left(z_{1}, \bar{z}_{1}\right) \mathscr{O}\left(z_{2}, \bar{z}_{2}\right)\right\rangle_{\lambda}=\left|z_{12}\right|^{-2 \Delta_{\lambda}}
$$

of a neutral and hermitian (for simplicity) operator $\mathscr{O}$ is still fixed by conformal symmetry, hence the effect of the marginal perturbation has to be a change of its conformal dimension. The $\lambda$ expansion of the functional integral gives

$$
\begin{aligned}
\left\langle\mathscr{O}\left(z_{1}, \bar{z}_{1}\right) \mathscr{O}\left(z_{2}, \bar{z}_{2}\right)\right\rangle_{\lambda}=\frac{1}{\left|z_{12}\right|^{2 \Delta}}[1 & +2 \pi C \lambda \log \frac{\left|z_{12}\right|}{\Lambda} \\
& \left.+\pi \lambda^{2} \log \frac{\left|z_{12}\right|}{\Lambda} \int d^{2} u G(u, \bar{u})+\mathrm{O}\left(\lambda^{3}\right)\right],
\end{aligned}
$$

where absence of a $\lambda$-index (e.g. in $\Delta$ ) always indicates evaluation in the free theory, and the objects in the r.h.s. are defined as follows. At first order, $C$ is the structure constant coming from the three-point function

$$
C=\left\langle\mathscr{O}(\infty) O_{[2]}^{(\mathrm{int})}(1) \mathscr{O}(0)\right\rangle .
$$

At second order, $G(u, \bar{u})$ is the undetermined part of the four-point function in terms of the anharmonic ratio $u \equiv\left(z_{12} z_{34}\right) /\left(z_{13} z_{24}\right)$,

$$
\left\langle\mathscr{O}\left(z_{1}, \bar{z}_{1}\right) O_{[2]}^{(\mathrm{int})}\left(z_{3}, \bar{z}_{3}\right) O_{[2]}^{(\mathrm{int})}\left(z_{4}, \bar{z}_{4}\right) \mathscr{O}\left(z_{2}, \bar{z}_{2}\right)\right\rangle=\frac{G(u, \bar{u})}{\left|z_{13}\right|^{2}\left|z_{32}\right|^{2}\left|z_{12}\right|^{2 \Delta-2}},
$$

and $\Lambda$ is a cutoff for the integral

$$
\int \frac{d^{2} z_{3}}{\left|z_{13}\right|^{2}\left|z_{32}\right|^{2}\left|z_{12}\right|^{2 \Delta-2}}=\frac{2 \pi}{\left|z_{12}\right|^{2 \Delta}} \log \frac{\left|z_{12}\right|}{\Lambda}, \quad \Lambda \ll 1 .
$$

The $\log \Lambda$ divergence in the two-point function requires the introduction of an appropriate regularization and a corresponding renormalization of the field $\mathscr{O}$. The logarithmic form of the divergent terms indeed has the effect of changing the exponent of the renormalized two-point function, thus changing $\Delta$. The operators we are interested in have a vanishing three-point function with $O_{[2]}^{\text {(int) }}$, i.e. $C=0$. The corrections in (3.4) therefore start at second order in $\lambda$, and the renormalized field is

where

$$
\mathscr{O}^{(\text {ren })}(z, \bar{z})=\Lambda^{\frac{1}{2} \pi \lambda^{2} J} \mathscr{O}(z, \bar{z}),
$$

We can see that

$$
\begin{aligned}
\left\langle\mathscr{O}^{(\text {ren })}\left(z_{1}, \bar{z}_{1}\right) \mathscr{O}^{(\text {ren })}\left(z_{2}, \bar{z}_{2}\right)\right\rangle_{\lambda} & =\Lambda^{\pi \lambda^{2} J}\left\langle\mathscr{O}\left(z_{1}, \bar{z}_{1}\right) \mathscr{O}\left(z_{2}, \bar{z}_{2}\right)\right\rangle_{\lambda} \\
& =\left(1+\pi \lambda^{2} J \log \Lambda+\cdots\right)\left|z_{12}\right|^{-2 \Delta}\left(1+\pi \lambda^{2} J \log \frac{\left|z_{12}\right|}{\Lambda}+\cdots\right) \\
& =\left(1+\pi \lambda^{2} J \log \left|z_{12}\right|+\cdots\right)\left|z_{12}\right|^{-2 \Delta} \\
& =\left|z_{12}\right|^{\pi \lambda^{2} J}\left|z_{12}\right|^{-2 \Delta}
\end{aligned}
$$


so the $\Lambda$-divergence is canceled, and the free-theory dimension $\Delta$ has flowed to a $\lambda$ dependent value

$$
\Delta_{\lambda}=\Delta-\frac{\pi}{2} \lambda^{2} J+\mathrm{O}\left(\lambda^{3}\right)
$$

The integral $J$ also gives the first-order $\lambda$-correction to the particular structure constant in (3.5). This can be seen from the functional integral expansion of the corresponding three-point function. For our case where the free-theory constant vanishes, we find

$$
C_{\lambda}=\lambda J+\mathrm{O}\left(\lambda^{2}\right)
$$

To compute the integral (3.9), we need to be able to calculate the four-point function (3.6) in the free orbifold theory. In the next section, we show how to do this.

\section{Four-point functions}

Our goal is to compute four-point functions ${ }^{3}$

$$
G(u, \bar{u})=\left\langle\mathscr{O}_{[n]}^{\dagger}(\infty) O_{[2]}^{(\mathrm{int})}(1) O_{[2]}^{(\mathrm{int})}(u, \bar{u}) \mathscr{O}_{[n]}(0)\right\rangle
$$

for primary operators $\mathscr{O}$ in the $n$-twisted sectors of the orbifold $\mathrm{SCFT}_{2}$. Some aspects of the computation are universal, depending only on the nature of the twists: we start by describing the covering surface appropriate to the twisted structure of (4.1); then we describe the stress-tensor method to compute the simplest four-point function with this structure, containing only bare twists. Finally, we turn to the cases containing interaction operators with $\mathscr{O}_{[n]}$ as the charged Ramond ground state, or as a bare twist field.

\subsection{The covering surface}

Twisted correlators such as (4.1) are complicated functions, with specific monodromies of their arguments fixed by their (bare) twist fields constituents. The standard way [12] of implementing the boundary conditions (2.6) for $G(u, \bar{u})$ is to map the 'base sphere' $S_{\text {base }}^{2}=\mathbb{C} \cup \infty$ to a ramified 'covering surface' $\Sigma_{\text {cover }}$, whose ramification points correspond to the position and the order of twists operators. At large $N$, the leading contribution comes from genus-zero covering surfaces. Denote coordinates on the base by $z \in S_{\text {base }}^{2}$, and coordinates on the covering sphere by $t \in S_{\text {cover }}^{2}$, and fix the four punctures on each surface to be

$$
\{z=0\} \mapsto\{t=0\},\{z=1\} \mapsto\left\{t=t_{1}\right\},\{z=u\} \mapsto\{t=x\},\{z=\infty\} \mapsto\{t=\infty\} .
$$

The method for finding $z(t)$ for generic monodromies was pioneered in [12] and generalized in [52]. For the specific monodromies (and topology) above,

$$
z(t)=\left(\frac{t}{t_{1}}\right)^{n}\left(\frac{t-t_{0}}{t_{1}-t_{0}}\right)\left(\frac{t_{1}-t_{\infty}}{t-t_{\infty}}\right) .
$$

\footnotetext{
${ }^{3} \mathrm{~A}$ note on convention: in this paper, fields inside correlation functions are to be understood in twodimensional theory, e.g. $\sigma_{n}(z, \bar{z})$, instead of $\sigma_{n}(z)$. However, when fixing a point in $\mathbb{C}^{2}$ we only write one argument for economy of notation. Thus, in (4.1), it should be understood that $\mathscr{O}_{[n]}(0)=\mathscr{O}_{[n]}(0, \overline{0})$, $O_{[2]}^{(\mathrm{int})}(1)=O_{[2]}^{(\mathrm{int})}(1, \overline{1})$, etc.
} 
The monodromies at $z=0$ and $z=\infty$ are evident, but at $z=1$ and $z=u$ they are implicit in the derivative $z^{\prime}(t)$, which must vanish at every branching point. Indeed,

$$
\frac{d z}{d t}=\frac{t_{1}-t_{\infty}}{t_{1}\left(t_{0}-t_{1}\right)} \frac{t^{n-1}}{\left(t-t_{\infty}\right)^{2}}\left[\left(t_{\infty}-t_{0}\right) t-n\left(t-t_{0}\right)\left(t-t_{\infty}\right)\right]
$$

vanishes at $t=0$ with the correct monodromy, while $x$ and $t_{1}$ must be the roots of the quadratic expression in brackets. This quadratic equation relates the parameters $t_{1}, t_{0}, t_{\infty}$ and $x$,

$$
x+t_{1}=\frac{n-1}{n} t_{0}+\frac{n+1}{n} t_{\infty} ; \quad x t_{1}=t_{0} t_{\infty} .
$$

We are free to choose one of the ratios $t_{0} / x, t_{0} / t_{1}, t_{\infty} / t_{1}$ and $t_{\infty} / x$ as long as they satisfy the two conditions (4.4), and we choose

$$
1-\frac{t_{0}}{x}=\frac{1}{x}, \quad \text { hence } \quad 1-\frac{t_{\infty}}{x}=\frac{1}{x+n}, \quad 1-\frac{t_{1}}{x}=\frac{2 x+n-1}{x(n+x)}
$$

which gives $u=z(x)$ as $[50,51]$

$$
u(x)=\frac{x^{n-1}(x+n)^{n+1}}{(x-1)^{n+1}(x+n-1)^{n-1}},
$$

a rational function.

\subsection{Four-point functions}

The covering map encodes the monodromies of functions like (4.1), with the twist structure

$$
g(u, \bar{u}) \equiv\left\langle\sigma_{n}(\infty) \sigma_{2}(1) \sigma_{2}(u, \bar{u}) \sigma_{n}(0)\right\rangle,
$$

into the ramification points of the covering surface. One way of computing $g(u, \bar{u})$, formulated by Lunin and Mathur [12], is to cut circles around the ramification points, replace them with vacua and compute the functional integral directly. An alternative ${ }^{4}[62]$ is to use the conformal Ward identity: if one is able to find the residue $r(u)$ of the following function on the base,

$$
f(z)=\frac{\left\langle T(z) \sigma_{n}(\infty) \sigma_{2}(1) \sigma_{2}(u, \bar{u}) \sigma_{n}(0)\right\rangle}{\left\langle\sigma_{n}(\infty) \sigma_{2}(1) \sigma_{2}(u, \bar{u}) \sigma_{n}(0)\right\rangle}=\frac{h}{(z-u)^{2}}+\frac{r(u)}{z-u}+\text { non-sing. }
$$

the Ward identity gives a differential equation

$$
\partial_{u} \log g(u)=r(u)
$$

which can be solved for the holomorphic part of $g(u, \bar{u})=g(u) \tilde{g}(\bar{u})$. The anti-holomorphic part $\tilde{g}(\bar{u})=\bar{g}(\bar{u})$ is obtained likewise, using $\tilde{T}(\bar{z})$.

In simpler orbifold theories, it is possible to find $r(u)$ by engineering the function with the appropriate poles and monodromies [62]. Here, we can follow refs. [16, 50-52] and use the covering surface as an aid, by computing the correlation functions on $S_{\text {cover }}^{2}$, where the

\footnotetext{
${ }^{4}$ Still other ways of computing general four-point functions $\left\langle\sigma_{m} \sigma_{n} \sigma_{p} \sigma_{q}\right\rangle$ have been recently given [35, 64].
} 
monodromies are trivial, and then mapping back: $f(z)$ is a function of the position of the stress tensor which, unlike the twists, is not placed on a branching/ramification point hence mapping from covering to base is just a conformal transformation. On the covering,

$$
f_{\text {cover }}(t)=\frac{\langle T(t) \mathbb{1}\rangle}{\langle\mathbb{1}\rangle}=0
$$

because the twists disappear, and when mapping back to base only the anomalous transformation of $T$ does not cancel in the fraction, so

$$
f(z)=\sum_{I}\left[\frac{c}{12}\left\{t_{I}, z\right\}+\left(\frac{d t_{I}}{d z}\right)^{2} f_{\text {cover }}\left(t_{I}(z)\right)\right]=\sum_{I} \frac{1}{2}\left\{t_{I}, z\right\} .
$$

The position of the twists appear as parameters implicit in the inverse maps $z \mapsto t$, which encode the twist structure of (4.7). There is a sum over $I$ in eq. (4.11) because $T(z)$ is a sum over copies (2.5). Around a branching point, there is one inverse map $t_{I}(z)$ for each copy entering the corresponding twist; at $z=u$, the insertion point of $\sigma_{2}$, there are two maps, which can be found locally $[17,51]$, as follows. Take the logarithm of the ratio $z(t) / z(x)$, i.e. $\log (z / u)=n \log \frac{t}{x}+\log \frac{t-t_{0}}{x-t_{0}}-\log \frac{t-t_{\infty}}{x-t_{\infty}}$, and expand both sides,

$$
\sum_{k=1}^{\infty} b_{k}(z-u)^{k}=(t-x)^{2} \sum_{k=0}^{\infty} a_{k}(t-x)^{k}, \quad \text { hence } t-x=\sum_{k=1}^{\infty} c_{k}(z-u)^{k / 2} .
$$

In the first equation, the coefficients are found from the Taylor expansions,

$$
b_{k}=\frac{(-1)^{k+1}}{k u^{k}}, \quad a_{k}=\frac{(-1)^{k+1}}{k+2}\left[\frac{1}{\left(x-t_{0}\right)^{k+2}}-\frac{1}{\left(x-t_{\infty}\right)^{k+2}}+\frac{n}{x^{k+2}}\right] .
$$

The coefficients $c_{k}$ are solved in terms of $a_{k}$ and $b_{k}$ order by order, by inserting the $c_{k}$ power series into the first equation in (4.12). The multiple inverses $z \mapsto t$ appear as multiple solutions for the $c_{k}$. After solving for the $c_{k}$, we can put the powers series into the r.h.s. of eq. (4.11), expand to order $(z-u)^{-1}$ and extract the desired residue. The coefficients $c_{1}, c_{2}$ and $c_{3}$ completely determine the result up to this order,

$$
c_{1}= \pm \sqrt{\frac{b_{1}}{a_{0}}}, \quad c_{2}=-\frac{a_{1} b_{1}}{2 a_{0}^{2}}, \quad c_{3}= \pm \frac{4 a_{0} a_{2} b_{1}^{2}-4 a_{0}^{3} b_{2}-5 a_{1}^{2} b_{1}^{2}}{8 a_{0}^{7 / 2} \sqrt{b_{1}}} .
$$

As expected, there are two solutions. When the parameters $t_{0}$ and $t_{\infty}$ in $a_{k}, b_{k}$ are written explicitly in terms of $x$, these coefficients are functions of $x$ alone, thus we find the residue $r$ as a function of $x$. One can check that $r(x)$ is the same for both choices of the $c_{k}$.

Solving eq. (4.9) requires expressing $r(x)$ as an explicit function of $u$, but there are multiple inverses of $u(x)$. It is easier to make a change of variables, and solve instead the differential equation

$$
\partial_{x} \log g(x)=u^{\prime}(x) r(x)
$$

whose solution is

$$
g(x)=c_{\sigma} \frac{x^{-\frac{2+5 n(n-1)}{8 n}}(x-1)^{\frac{2+5 n(n+1)}{8 n}}(x+n)^{\frac{2-n(n+1)}{8 n}}(x+n-1)^{-\frac{2-n(n-1)}{8 n}}}{\left(x+\frac{n-1}{2}\right)^{1 / 4}} .
$$


The integration constant $c_{\sigma}$ has to be determined by looking at OPE limits (see appendix C)..$^{5}$

Now, we have found a function parameterized by the pre-image of $u$ under the covering map $z(t)$. For fixed $u=u_{*}$, there are $\mathbf{H}$ different pre-images $x_{\mathfrak{a}}, \mathfrak{a}=\mathbf{1}, \cdots, \mathbf{H}$, solutions of the equation

$$
x^{n-1}(x+n)^{n+1}-u_{*}(x-1)^{n+1}(x+n-1)^{n-1}=0 .
$$

The degree of the polynomial shows that $\mathbf{H}=2 n$. Note that this is not the number of sheets of the ramified covering ( $u$ is the position of a branching point), it is the number of different covering maps with the assumed monodromy conditions; $\mathbf{H}$ is a Hurwitz number $[16,17,52]$.

The method has thus yielded $\mathbf{H}$ functions $g\left(x_{\mathfrak{a}}(u)\right)$. This was expected, because the $S_{N}$ structure of the composition of cycles in eq. (4.7) is not completely fixed. Labeling cycles by the position of their twists operators, those entering $g(u, \bar{u})$ must compose to the identity,

$$
(n)_{\infty}(2)_{1}(2)_{u}(n)_{0}=1,
$$

otherwise the correlator vanishes. There are several collections $\left\{(n)_{\infty},(2)_{1},(2)_{u},(n)_{0}\right\}$ of cycles which solve eq. (4.18), ${ }^{6}$ and these collections can be arranged into equivalence classes defined by

$$
(n)_{\infty}(2)_{1}(2)_{u}(n)_{0} \sim h(n)_{\infty} h^{-1} h(2)_{1} h^{-1} h(2)_{u} h^{-1} h(n)_{0} h^{-1} \quad \forall \quad h \in S_{N} .
$$

The existence of different such equivalence classes is the reason for the existence of different functions $g\left(x_{\mathfrak{a}}(u)\right)$; there are precisely $\mathbf{H}=2 n$ equivalence classes [52]. Inside each of these classes, let $C_{\mathbf{s}}(N)$ be the number of collections $\left\{(n)_{\infty},(2)_{1},(2)_{u},(n)_{0}\right\}$ for which the cycles involve a fixed number $\mathbf{s}$ of distinct elements of $\{1,2, \cdots, N\}$. Then it can be shown [52] that $C_{\mathbf{s}}$ is the same for all classes, and that, for large $N$, it scales as

$$
C_{\mathbf{s}}=N^{\mathbf{s}-\frac{1}{2} \sum_{r=1}^{4} n_{r}}\left[\varpi\left(n_{r}\right)+\mathrm{O}(1 / N)\right],
$$

where $n_{1}=n=n_{4}$ and $n_{2}=2=n_{3}$ are the order of the $q=4$ twists involved in (4.7). But $n_{r}-1$ is also the order of the ramification points of the covering surface, $\mathbf{s}$ is the number of its sheets, hence its genus is fixed by the Riemann-Hurwitz formula

$$
\mathbf{g}=1-\mathbf{s}+\frac{1}{2} \sum_{r=1}^{q}\left(n_{r}-1\right) .
$$

We thus see that $C_{\mathbf{s}}(N) \equiv C_{\mathbf{g}}(N) \sim N^{-\mathbf{g}-1}$, therefore the covering surface with $\mathbf{g}=0$ constructed in section 4.1 gives the leading contribution at large $N$ [12]. For our four-point functions, the Riemann-Hurwitz formula gives $\mathbf{s}=-\mathbf{g}+n+1$, hence we see that, for the covering surface to have genus zero, we must have

$$
1 \leq n<N .
$$

\footnotetext{
${ }^{5}$ We emphasize that the function $\left\langle\sigma_{n}(\infty) \sigma_{2}(1) \sigma_{2}(u, \bar{u}) \sigma_{n}(0)\right\rangle$ is known in the literature, calculated by other methods. Our point is to take it as an instructive example of the specific method we use.

${ }^{6}$ The total number of such solutions can be found with Frobenius' formula [65].
} 
When we sum over the orbits of individual cycles to make an $S_{N}$-invariant correlation function, we get all terms in each of the equivalence classes above,

$$
\left\langle\sigma_{[n]}(\infty) \sigma_{[2]}(1) \sigma_{[2]}(u, \bar{u}) \sigma_{[n]}(0)\right\rangle=\frac{\varpi(n)}{N} \sum_{\mathfrak{a}=1}^{\mathbf{H}} g\left(x_{\mathfrak{a}}(u)\right) \bar{g}\left(\bar{x}_{\mathfrak{a}}(\bar{u})\right) .
$$

This sum corresponds to different OPE channels resulting from composing the twist permutations, not only for $g(u, \bar{u})$ but for the other functions $G(u, \bar{u})$ which share the same twist structure.

\subsubsection{Charged Ramond fields}

Let us now turn to the function

$$
G_{R}(u, \bar{u})=\left\langle R_{[n]}^{-}(\infty) O_{[2]}^{(\mathrm{int})}(1) O_{[2]}^{(\mathrm{int})}(u, \bar{u}) R_{[n]}^{+}(0)\right\rangle .
$$

The Ramond fields $R_{[n]}^{ \pm}(z, \bar{z})$ are lifted to the corresponding spin field $S^{ \pm}(t, \bar{t})$, so we compute

$$
F_{\text {cover }}(t)=\frac{\left\langle T(t) S^{-}(\infty) O^{(\text {int })}\left(t_{1}, \bar{t}_{1}\right) O^{(\mathrm{int})}(x, \bar{x}) S^{+}(0)\right\rangle}{\left\langle S^{-}(\infty) O^{(\mathrm{int})}\left(t_{1}, \bar{t}_{1}\right) O^{\text {(int) }}(x, \bar{x}) S^{+}(0)\right\rangle}
$$

and then find the residue $H$ of the function

$$
\begin{aligned}
F(z) & =\frac{\left\langle T(z) R_{[n]}^{-}(\infty) O_{[2]}^{(\mathrm{int})}(1) O_{[2]}^{(\mathrm{int})}(u, \bar{u}) R_{[n]}^{+}(0)\right\rangle}{\left\langle R_{[n]}^{-}(\infty) O_{[2]}^{(\mathrm{int})}(1) O_{[2]}^{(\mathrm{int})}(u, \bar{u}) R_{[n]}^{+}(0)\right\rangle} \\
& =2\left[\frac{1}{2}\{t, z\}+\left(\frac{d t}{d z}\right)^{2} F_{\text {cover }}(t(z), x)\right]=\frac{H(x)}{z-u}+\cdots
\end{aligned}
$$

with $t(z)$ one of the maps obtained from eqs. (4.12) and (4.14).

The deformation operator, denoted by $O^{(\text {int })}(t, \bar{t})$ - without a twist index since there are no twists on the covering surface - can be expressed on $S_{\text {cover }}^{2}$ in terms of the basic fields only, because the contour integrals in the super-current modes $G_{-\frac{1}{2}}^{\alpha A}=\frac{1}{2 \pi i} \oint d z G^{\alpha A}(z)$ just pick up a residue (see e.g. [18]). The result is a sum of products of bosonic currents, free fermions and spin fields coming from the lifting of the NS chiral field $O_{[2]}^{(0,0)}(z, \bar{z}) \mapsto$ $S^{+}(t) \tilde{S}^{\dot{+}}(\bar{t})$. Writing spin fields as exponentials,

$$
\begin{aligned}
O^{(\mathrm{int})}=a_{\text {int }}[ & : \partial X^{\mathrm{i} 1} e^{+\frac{i}{2}\left(\phi_{1}+\phi_{2}\right)}\left(\bar{\partial} X^{\mathrm{i} 2} e^{+\frac{i}{2}\left(\tilde{\phi}_{1}+\tilde{\phi}_{2}\right)}-\left(\bar{\partial} X^{\mathrm{i} 1}\right)^{\dagger} e^{-\frac{i}{2}\left(\tilde{\phi}_{1}+\tilde{\phi}_{2}\right)}\right): \\
& -: \partial X^{\mathrm{i} 2} e^{+\frac{i}{2}\left(\phi_{1}+\phi_{2}\right)}\left(\left(\bar{\partial} X^{\mathrm{i} 2}\right)^{\dagger} e^{-\frac{i}{2}\left(\tilde{\phi}_{1}+\tilde{\phi}_{2}\right)}+\bar{\partial} X^{\mathrm{i} 1} e^{+\frac{i}{2}\left(\tilde{\phi}_{1}+\tilde{\phi}_{2}\right)}\right): \\
& +:\left(\partial X^{\mathrm{i} 1}\right)^{\dagger} e^{-\frac{i}{2}\left(\phi_{1}+\phi_{2}\right)}\left(\left(\bar{\partial} X^{\mathrm{i} 2}\right)^{\dagger} e^{-\frac{i}{2}\left(\tilde{\phi}_{1}+\tilde{\phi}_{2}\right)}+\bar{\partial} X^{\mathrm{i} 1} e^{+\frac{i}{2}\left(\tilde{\phi}_{1}+\tilde{\phi}_{2}\right)}\right): \\
& \left.+:\left(\partial X^{\mathrm{i} 2}\right)^{\dagger} e^{-\frac{i}{2}\left(\phi_{1}+\phi_{2}\right)}\left(\bar{\partial} X^{\mathrm{i} 2} e^{+\frac{i}{2}\left(\tilde{\phi}_{1}+\tilde{\phi}_{2}\right)}-\left(\bar{\partial} X^{\mathrm{i} 1}\right)^{\dagger} e^{-\frac{i}{2}\left(\tilde{\phi}_{1}+\tilde{\phi}_{2}\right)}\right):\right] .
\end{aligned}
$$

The constant $a_{\text {int }}$ can be conveniently chosen by a redefinition of the deformation parameter $\lambda$. For now, we leave it unspecified. To compute the correlators, the strategy is to show that contractions of $T(t)$ with the fields in the numerator of (4.25) are always proportional 
to $\mathcal{G}=\left\langle S^{-}(\infty) O^{(\mathrm{int})}\left(t_{1}, \bar{t}_{1}\right) O^{(\mathrm{int})}(x, \bar{x}) S^{+}(0)\right\rangle$, appearing in the denominator of eq. (4.25). We can decompose $T(t)=T_{B}(t)+T_{F}(t)$ into bosonic and fermionic parts, respectively

$$
\begin{aligned}
& T_{B}(t)=-\frac{1}{4} \epsilon_{\dot{A} \dot{B}} \epsilon_{A B}: \partial X^{\dot{A} A}(t) \partial X^{\dot{B} B}(t): \\
& T_{F}(t)=-\frac{1}{2}:\left[\partial \phi_{1}(t) \partial \phi_{1}(t)+\partial \phi_{2}(t) \partial \phi_{2}(t)\right]:
\end{aligned}
$$

As far as bosons are concerned, each term of the product $O^{(\mathrm{int})}\left(t_{1}\right) O^{(\mathrm{int})}(x)$ has the structure $\partial X^{\dot{C} C}\left(t_{1}\right) \partial X^{\dot{E} E}(x)$ multiplied by "transparent" fermionic or anti-holomorphic factors. Using the conformal Ward identity and the two-point functions (A.5),

$$
\begin{aligned}
\left\langle T_{B}(t) \partial X^{\dot{C} C}\left(t_{1}\right) \partial X^{\dot{E} E}(x)\right\rangle & =\left[\frac{\partial_{t_{1}}}{t-t_{1}}+\frac{\partial_{x}}{t-x}+\frac{1}{\left(t-t_{1}\right)^{2}}+\frac{1}{(t-x)^{2}}\right] \frac{2 \epsilon^{\dot{C}} \dot{E} \epsilon E}{\left(t_{1}-x\right)^{2}} \\
& =\frac{\left(t_{1}-x\right)^{2}}{\left(t-t_{1}\right)^{2}(t-x)^{2}}\left\langle\partial X^{\dot{C} C}\left(t_{1}\right) \partial X^{\dot{E} E}(x)\right\rangle .
\end{aligned}
$$

Hence we can recompose $\mathcal{G}$, and obtain

$$
\frac{\left\langle T_{B}(t) S^{-}(\infty) O^{(\mathrm{int})}\left(t_{1}, \bar{t}_{1}\right) O^{(\mathrm{int})}(x, \bar{x}) S^{+}(0)\right\rangle}{\left\langle S^{-}(\infty) O^{\text {(int) }}\left(t_{1}, \bar{t}_{1}\right) O^{\text {(int) }}(x, \bar{x}) S^{+}(0)\right\rangle}=\frac{\left(t_{1}-x\right)^{2}}{\left(t-t_{1}\right)^{2}(t-x)^{2}} .
$$

For the fermionic part of the calculation, it is very helpful to organize $O^{(\text {int) }}$ as

where

$$
O^{(\mathrm{int})}(t, \bar{t}) \equiv V_{-}(t, \bar{t})+V_{+}(t, \bar{t}),
$$

the (a.h)s being combinations of anti-holomorphic fields which can be read from (4.27). This makes it is clear that contractions with $O_{[2]}^{(\text {int })}$ are very simple, and

$$
\begin{aligned}
& \sum_{r} \lim _{v \rightarrow t}\left\langle\partial \phi_{r}(v) \partial \phi_{r}(t) S^{-}(\infty) O^{(\mathrm{int})}\left(t_{1}\right) O^{(\mathrm{int})}(x, \bar{x}) S^{+}(0)\right\rangle-\sum_{r} \lim \phi_{r}(t) \phi_{r}(v) \\
& =\left[\frac{(i / 2)^{2}}{t^{2}}+\frac{(i / 2)^{2}}{\left(t-t_{1}\right)^{2}}+\frac{(i / 2)^{2}}{(t-x)^{2}}\right] \mathcal{G} \\
& \quad+\frac{2(i / 2)^{2}}{\left(t-t_{1}\right)(t-x)}\left\langle S^{-}(\infty)\left[V_{-}\left(t_{1}\right)-V_{+}\left(t_{1}\right)\right]\left[V_{-}(x)-V_{+}(x)\right] S^{+}(0)\right\rangle
\end{aligned}
$$

The second line in the r.h.s. can be further simplified because, since the only nonvanishing two-point functions (A.7) are between a field and its conjugate, it follows that $\left\langle V_{ \pm}(t, \bar{t}) V_{ \pm}(v, \bar{v})\right\rangle=0$, hence

$$
\left\langle S^{-}(\infty)\left[V_{-}\left(t_{1}\right)-V_{+}\left(t_{1}\right)\right]\left[V_{-}(x)-V_{+}(x)\right] S^{+}(0)\right\rangle=-\mathcal{G} .
$$

Putting this back in (4.30), $\mathcal{G}$ appears as a common factor canceled in (4.25),

$$
\frac{\left\langle T_{F}(t) S^{-}(\infty) O^{(\mathrm{int})}\left(t_{1}, \bar{t}_{1}\right) O^{(\mathrm{int})}(x, \bar{x}) S^{+}(0)\right\rangle}{\left\langle S^{-}(\infty) O^{(\mathrm{int})}\left(t_{1}, \bar{t}_{1}\right) O^{(\mathrm{int})}(x, \bar{x}) S^{+}(0)\right\rangle}=\frac{1}{4}\left[\frac{1}{t^{2}}+\left(\frac{1}{t-t_{1}}-\frac{1}{t-x}\right)^{2}\right] .
$$


Combining (4.28) and (4.32), we get

$$
F_{\text {cover }}(t)=\frac{\left(t_{1}-x\right)^{2}}{\left(t-t_{1}\right)^{2}(t-x)^{2}}+\frac{1}{4}\left[\frac{1}{t^{2}}+\left(\frac{1}{t-t_{1}}-\frac{1}{t-x}\right)^{2}\right] .
$$

Inverting the maps, we find the residue $H(x)$ of $F(z)$ to be

$$
\begin{aligned}
H(x)=-[ & 16 x^{4}+32(2 n-1) x^{3} \\
& +4(2 n-1)(10 n-7) x^{2} \\
& +4(n-1)[10(n-1) n+3] x \\
& \left.+5(n-2)(n-1)^{2} n\right]\left[4 n(n+2 x-1)^{3}\right]^{-1} .
\end{aligned}
$$

The solution of the differential equation $\partial_{x} \log G_{R}(x)=u^{\prime}(x) H(x)$ is now easily found,

$$
G_{R}(x)=C_{R} \frac{x^{\frac{5(2-n)}{4}}(x-1)^{\frac{5(2+n)}{4}}(x+n)^{\frac{2-3 n}{4}}(x+n-1)^{\frac{2+3 n}{4}}}{\left(x+\frac{n-1}{2}\right)^{4}} .
$$

where $C_{R}$ is an integration constant.

\subsubsection{Bare twists}

Let us also consider

$$
G_{\sigma}(u, \bar{u})=\left\langle\sigma_{[n]}(\infty) O_{[2]}^{(\mathrm{int})}(1) O_{[2]}^{(\mathrm{int})}(u, \bar{u}) \sigma_{[n]}(0)\right\rangle
$$

appearing in the second-order correction of the two-point function of bare twist fields. The computation of

$$
\begin{aligned}
F_{\text {cover }}(t) & =\frac{\left\langle T(t) \mathbb{1}(\infty) O^{(\mathrm{int})}\left(t_{1}, \bar{t}_{1}\right) O^{(\mathrm{int})}(x, \bar{x}) \mathbb{1}(0)\right\rangle}{\left\langle\mathbb{1}(\infty) O^{(\mathrm{int})}\left(t_{1}, \bar{t}_{1}\right) O^{(\mathrm{int})}(x, \bar{x}) \mathbb{1}(0)\right\rangle} \\
& =\frac{\left(t_{1}-x\right)^{2}}{\left(t-t_{1}\right)^{2}(t-x)^{2}}+\frac{1}{4}\left(\frac{1}{t-t_{1}}-\frac{1}{t-x}\right)^{2},
\end{aligned}
$$

goes as before (but is simpler), and we find

$$
G_{\sigma}(x)=C_{\sigma} \frac{x^{-\frac{1-10 n+5 n^{2}}{4 n}}(x-1)^{\frac{1+10 n+5 n^{2}}{4 n}}(x+n)^{\frac{1+2 n-3 n^{2}}{4 n}}(x+n-1)^{-\frac{1-2 n-3 n^{2}}{4 n}}}{\left(x+\frac{n-1}{2}\right)^{4}} .
$$

where $C_{\sigma}$ is an integration constant. The same function has been computed in appendix $\mathrm{E}$ of ref. [17], but using a different parameterization map $u(x)$, in place of (4.6) (hence their function $G(x)$ is different from ours).

\section{OPE limits, fusion rules and structure constants}

The short-distance behavior of $G(u, \bar{u})$ in the limits $u \rightarrow 1,0, \infty$ contains the complete conformal data of the operator product expansions of the fields involved - i.e. the OPE fusion rules. Recall that super-conformal invariance fixes the form of the OPE algebra of generic primary holomorphic fields $\mathscr{O}_{k}^{\left(j_{k}^{3}\right)}(u)$ with dimensions $\Delta_{k}$ and R-charges $j_{k}^{3}$ to be

$$
\mathscr{O}_{1}^{\left(j_{1}^{3}\right)}(u, \bar{u}) \mathscr{O}_{2}^{\left(j_{2}^{3}\right)}(0)=\sum_{k} C_{12 k}|u|^{\Delta_{k}-\Delta_{1}-\Delta_{2}} \mathscr{O}_{k}^{\left(j_{k}^{3}\right)}(0)+\text { descendants },
$$

with structure constants $C_{12 k}$, and $j_{k}^{3}=j_{1}^{3}+j_{2}^{3}$. 


\subsection{The OPE of two interaction operators}

The OPE of two interaction operators appears in the limit $u \rightarrow 1$ of $G(u, \bar{u})$. To extract this limit from $G(x)$, we have to find the inverse maps $x_{\mathfrak{a}}(u)$ which contribute to the singularities near $u=1$. For both $G_{R}(x)$ and $G_{\sigma}(x)$, there are clearly only two contributions, i.e. limits where $G(x)$ becomes singular, namely: ${ }^{7} x=\infty$ and $x=\frac{1-n}{2}$, the former with multiplicity one, and the latter with multiplicity three. We label the two corresponding functions, given in (B.5), as $x_{\mathfrak{a}}^{1}(u)$, with a (gothic) index $\mathfrak{a}=1,2$, and the superscript indicating that $u \rightarrow 1$. Each function gives a channel of the fusion rule, according to eq. (4.23). Both functions $G_{R}(x)$ and $G_{\sigma}(x)$ have the same behavior in these limits, as it was necessary for consistency, since both functions should give the same OPE $\left[O_{[2]}^{(\mathrm{int})}\right] \times\left[O_{[2]}^{(\mathrm{int})}\right]=[\mathbb{1}]+\left[\sigma_{[3]}\right]$, where the r.h.s. is based on the composition of permutations. We mostly focus on $G_{R}(x)$ in what follows, similar calculations for $G_{\sigma}(x)$ are listed in appendix C.

Determining the constants of integration. For $x \rightarrow \infty, G_{R}(x) \approx C_{R} x^{2}$. Inserting $x=x_{1}^{1}(u)$ given by eq. (B.5), we obtain

$$
G_{R}\left(x_{1}^{1}(u)\right)=C_{R} \frac{16 n^{2}}{(1-u)^{2}}+0 \times \frac{1}{1-u}+\text { non-singular }
$$

By formula (5.1), since $O_{[2]}^{(\mathrm{int})}$ has weight $h_{\mathrm{int}}=1$, the leading singular term shows an operator of dimension $h=2-2=0$ - the identity operator. Also, the coefficient of the term $\sim(1-u)^{-1}$ is zero, hence there is no contribution from a field of dimension $h=1$, as it was to be expected for a truly marginal deformation.

The function in eq. (5.2) corresponds to a correlator where the permutations in the twists form one representative element of the equivalence class where the 2-cycles of the interaction operators cancel. This happens when they share both elements. At order $N^{-1}$, there must be $\mathbf{s}=n+1$ elements entering the permutation, cf. eq. (4.20), so we can take this representative function to be

$$
\left\langle R_{(1, \cdots, n)}^{-}(\infty) O_{(1, n+1)}^{\text {(int })}(1) O_{(1, n+1)}^{\text {(int })}(u, \bar{u}) R_{(n, \cdots, 1)}^{+}(0)\right\rangle=G_{R}\left(x_{1}^{1}(u)\right) G_{R}\left(x_{1}^{1}(\bar{u})\right)
$$

or any other with a global relabeling of elements in the cycles.

We now fix the constant $a_{\text {int }}$ in (4.27) so that the non- $S_{N}$-invariant two-point functions are normalized,

$$
\left\langle O_{2}^{(\mathrm{int})}(\infty) O_{2}^{(\mathrm{int})}(1)\right\rangle=1 .
$$

Note that in these functions the two-cycles must share both of their elements, since, as in eq. (4.18), we must have $(2)_{\infty}(2)_{1}=1$. With this definition, the normalized $S_{N}$-invariant operator is

$$
O_{[2]}^{(\mathrm{int})}(z, \bar{z})=\frac{1}{\mathscr{S}_{2}(N)} \sum_{h \in S_{N}} O_{h^{-1}(12) h}^{(\mathrm{int})}(z, \bar{z}) .
$$

\footnotetext{
${ }^{7}$ These correspond to the solutions of $t_{1}(x)=x$. Fortunately, we do not need to find the other solutions of the $2 n$ th-order polynomial equation (4.17) for $u_{*}=1$.
} 
Together with the normalization (2.13), inserting the limit (5.2), back into the four-point function (5.3) we find $16 n^{2} C_{R}=1$. The same reasoning can be applied to the function $G_{\sigma}(x)$, which has the exact same limit as (5.2) in this channel. Therefore

$$
C_{R}=\frac{1}{16 n^{2}}=C_{\sigma}
$$

With the functions $G(x)$ completely fixed, we can now look at other OPEs and derive structure constants.

The $\sigma_{3}$ channel. In the other channel corresponding to $u \rightarrow 1$, we must expand $G_{R}(x)$ around $x=\frac{1-n}{2}$, and insert $x_{2}^{1}(u)$ given by eq. (B.5),

$$
\begin{aligned}
G_{R}\left(x_{2}^{1}(u)\right)= & -\frac{4\left(n^{2}-1\right)^{\frac{1}{3}}\left(\frac{n+1}{n-1}\right)^{\frac{n}{2}}\left(\frac{1}{3} n\right)^{\frac{4}{3}} C_{R}}{(1-u)^{4 / 3}} \\
& +\frac{\frac{1}{5}\left(7+2 n^{2}\right)\left(\frac{n^{2}}{9\left(n^{2}-1\right)}\right)^{\frac{1}{3}}\left(\frac{n+1}{n-1}\right)^{\frac{n}{2}} C_{R}}{(1-u)^{2 / 3}} \\
& -\frac{4 \cdot 3^{-\frac{7}{3}}\left(\frac{n+1}{n-1}\right)^{\frac{n}{2}}\left(n^{2}-1\right)^{\frac{1}{3}} n^{4} C_{R}}{(1-u)^{1 / 3}}+\text { non-singular }
\end{aligned}
$$

Once again, the coefficient of next-to-leading divergence, $\sim(1-u)^{3 / 3}$, vanishes, showing that there is no dimension-one operator in this conformal family either. The leading singularity shows the presence of an operator of dimension $\frac{2}{3}=h_{3}^{\sigma}$, so we have found $\sigma_{3}$ itself, and the OPE

$$
O_{2}^{(\mathrm{int})}(u, \bar{u}) O_{2}^{(\mathrm{int})}(1)=\frac{\left\langle O_{2}^{(\mathrm{int})}(\infty) \sigma_{3}(1) O_{2}^{(\mathrm{int})}(0)\right\rangle}{|1-u|^{8 / 3}} \sigma_{3}(1)+\cdots
$$

whose structure constant is given in eq. (C.5), and found independently from $G_{\sigma}(x)$. Inserting the OPE into the correlation function we find the structure constant

$$
C_{n 3 n}^{R^{-} \sigma R^{+}} \equiv\left\langle R_{n}^{-}(\infty) \sigma_{3}(1) R_{n}^{+}(0)\right\rangle
$$

involving non- $S_{N}$-invariant Ramond fields and one three-twist. The leading term in eq. (5.7) gives us

$$
\log C_{n 3 n}^{R^{-} \sigma R^{+}}=\left(n+\frac{2}{3}\right) \log (n+1)-\left(n-\frac{2}{3}\right) \log (n-1)-\frac{4}{3} \log n+\frac{4}{3} \log 3+\frac{1}{3} \log 2
$$

after taking eq. (C.5) into account.

The correlation function that gives eq. (5.7) lies in an equivalence class where the 2cycles of the interaction operator share only one element, thus forming $\sigma_{3}$. The multiplicity 3 of the solution $x=\frac{1-n}{2}$ for eq. (4.17) implies there are three different equivalence classes 
with this property. Representative functions for each of those classes are ${ }^{8}$

$$
\begin{aligned}
G_{R}( & \left.x_{2}^{1}(u)\right) G_{R}\left(x_{2}^{1}(\bar{u})\right) \\
& =\left\langle R_{(1,2, \cdots, n-1, n)}^{-}(\infty) O_{(1, n+1)}^{(\mathrm{int})}(u, \bar{u}) O_{(1,2)}^{(\mathrm{int})}(1) R_{(n+1, n, n-1, \cdots, 2)}^{+}(0)\right\rangle \\
& =\left\langle R_{(1,2, \cdots, n-1, n)}^{-}(\infty) O_{(2, n+1)}^{(\mathrm{int})}(u, \bar{u}) O_{(1, n+1)}^{(\mathrm{int})}(1) R_{(n+1, n, n-1, \cdots, 2)}^{+}(0)\right\rangle \\
& =\left\langle R_{(1,2, \cdots, n-1, n)}^{-}(\infty) O_{(1,2)}^{(\mathrm{int})}(u, \bar{u}) O_{(2, n+1)}^{(\mathrm{int})}(1) R_{(n+1, n, n-1, \cdots, 2)}^{+}(0)\right\rangle
\end{aligned}
$$

One can check that the permutations do satisfy eq. (4.18). Note that, by necessity, the twists in the Ramond fields are not the inverse of one another, so the two-point function

$$
\left\langle R_{(1,2, \cdots, n-1, n)}^{-}(\infty) R_{(n+1, n, n-1, \cdots, 2)}^{+}(0)\right\rangle=0 .
$$

Thus we see that the $\sigma_{3}$ channel of the fusion $\left[O_{[2]}^{(\mathrm{int})}\right] \times\left[O_{[2]}^{(\mathrm{int})}\right]$ is always present, because the interaction operator is necessarily an $S_{N}$-invariant object, but eqs. (5.11) and (5.12) mean that $\sigma_{3}$ does not contribute to the correction of the two-point functions of individual, non- $S_{N}$-invariant Ramond fields $R_{n}^{ \pm}$. It only contributes to the $S_{N}$-invariant combination $R_{[n]}^{ \pm}$, by weaving together different individual terms.

The OPE $\boldsymbol{R}_{n}^{-} \boldsymbol{R}_{n}^{+}$. Although the positions of the Ramond fields are fixed in (4.23), we can loosen the punctures back to eq. (3.6), fix them differently as $z_{2}=\infty, z_{3}=0, z_{4}=1$, in which case $z_{1}=u$, to find

$$
\left\langle O_{[2]}^{(\mathrm{int})}(\infty) R_{[n]}^{-}(u, \bar{u}) O_{[2]}^{(\mathrm{int})}(0) R_{[n]}^{+}(1)\right\rangle=|1-u|^{4-n} G_{R}(u, \bar{u}) .
$$

Now the limit $u \rightarrow 1$ corresponds to the $\mathrm{OPE} R_{[n]}^{-}(u, \bar{u}) R_{[n]}^{+}(1)$. The expansion near $u=1$ for channel (5.2) is

$$
\left\langle O_{2}^{(\mathrm{int})}(\infty) R_{n}^{-}(u, \bar{u}) O_{2}^{(\mathrm{int})}(0) R_{n}^{+}(1)\right\rangle=\frac{1}{|1-u|^{n}}+\cdots
$$

This corresponds to an operator of dimension zero, and is in fact the correct expression for the two-point function of Ramond fields, eq. (2.13). In the channel (5.7) we now find the behavior $\sim(1-u)^{-n+\frac{8}{3}}$, indicating a twist-three operator of holomorphic weight

$$
h=\frac{n+4}{2}+h_{3}^{\sigma} .
$$

To understand the appearance of $\sigma_{3}$ in a channel of the OPE $R_{n}^{-} R_{n}^{+}$, let us consider the simpler case of the correlator with bare twists only. Changing the points of eq. (4.7), we can find the OPE $\sigma_{n} \sigma_{n}$ from the limit $u \rightarrow 1$ of the function

$$
\left\langle\sigma_{2}(\infty) \sigma_{n}(1) \sigma_{n}(u, \bar{u}) \sigma_{2}(0)\right\rangle=|1-u|^{4\left(h_{2}^{\sigma}-h_{n}^{\sigma}\right)} g(u, \bar{u}) .
$$

\footnotetext{
${ }^{8}$ An elegant and useful way of describing the different classes of permutations with the correct cycle structure and which satisfy eq. (4.18) is given in refs. [17, 52] in terms of inequivalent diagrams. The permutations in eqs. (5.11a)-(5.11c) correspond, respectively, to the following diagrams in ref. [17]: a) the top diagram of figure 4 ; b) the second diagram in figure 4 ; c) the top diagram of figure 5 .
} 
Channel (C.6) gives an operator of dimension zero, and channel (C.7) an operator of dimension $\frac{2}{3}=h_{3}^{\sigma}$. This gives us the fusion rule

$$
\left[\sigma_{n}\right] \times\left[\sigma_{n}\right]=[\mathbb{1}]+\left[\sigma_{3}\right]+\cdots
$$

Of course, there are other twists in the r.h.s. but they cannot be found from the fourpoint function we have began with, because of the condition (4.18). As discussed above, in channel (C.6) the two twists $\sigma_{2}$ in the correlator have inverse cycles, hence it is necessary that the two twists $\sigma_{n}$ also be the inverse of each other; this gives $\mathbb{1}$ in the fusion rule. As for the channel (C.7), we have seen that the cycles in $\sigma_{2}$ then only have one overlapping element, say, $\sigma_{(k \ell)} \sigma_{(k m)}=\sigma_{(k \ell m)}$. Hence for eq. (4.18) to be satisfied the two $\sigma_{n}$ operators must compose to $\sigma_{n} \sigma_{n}=\sigma_{(m \ell k)}$, which is why $\sigma_{3}$ appears.

\subsection{Non-BPS operators in the OPEs of $R_{n}^{ \pm}$with $O_{2}^{(i n t)}$}

We now turn to the limit $u \rightarrow 0$, where the interaction operator collides with either the Ramond field $R_{n}^{+}(0)$ or with the bare twisted field $\sigma_{n}(0)$, depending on the function we analyze, if either $G_{R}$ or $G_{\sigma}$. Now one can find all $2 n$ solutions of eq. (4.17), viz. $x=0$ (with multiplicity $n-1$ ) and $x=-n$ (with multiplicity $n+1$ ), all contributing to the OPE limits.

The function $G_{R}(u, \bar{u})$ gives the OPE $O_{[2]}^{(\text {int })}(u, \bar{u}) R_{[n]}^{+}(0)$. Using (B.1),

$$
\begin{aligned}
& G_{R}\left(x_{1}^{0}(u)\right)=u^{-\frac{5}{4} \frac{n-2}{n-1}}\left(C_{1}+c_{1} u^{\frac{1}{n-1}}+\cdots\right), \\
& G_{R}\left(x_{2}^{0}(u)\right)=u^{-\frac{1}{4} \frac{3 n-2}{n+1}}\left(C_{2}+c_{2} u^{\frac{1}{n+1}}+\cdots\right) .
\end{aligned}
$$

Counting powers of $u$, we find that the OPE $O_{2}^{(\text {int })} R_{n}^{+}$results in a twisted field $Y_{m}^{+}$which is positively R-charged with $j^{3}=\frac{1}{2}$, and has the holomorphic dimension

$$
h_{m}^{Y}=\frac{3}{2 m}+h_{m}^{\sigma} .
$$

Channels $x_{1}^{0}(u)$ and $x_{2}^{0}(u)$ give $m=n-1$ and $m=n+1$, respectively.

The OPE $O_{[2]}^{(\text {int) }} R_{n}^{-}$, is obtained in the limit $u \rightarrow 0$ of

$$
\left\langle O_{[2]}^{(\mathrm{int})}(\infty) R_{[n]}^{-}(u, \bar{u}) O_{[2]}^{(\mathrm{int})}(0) R_{[n]}^{+}(1)\right\rangle=|1-u|^{4-n} G_{R}(u, \bar{u}),
$$

which follows from the same procedure of fixing points used to find (5.13). Since the factor of $(1-u)^{\frac{4-n}{2}}$ does not contribute to the leading term near $u=0$, we immediately find the same expansion as before. Now the resulting fields $Y_{m}^{-}$have the same dimensions (5.20), but opposite R-charge, $j^{3}=-\frac{1}{2}$.

In summary, we have found the fusion rules

$$
\left[O_{[2]}^{(\mathrm{int})}\right] \times\left[R_{[n]}^{ \pm}\right]=\left[Y_{[n-1]}^{ \pm}\right]+\left[Y_{[n+1]}^{ \pm}\right],
$$

where the fields $Y_{m}^{ \pm}$have the dimension (5.20). The appearance of $m=n \pm 1$ in the r.h.s. is a basic consequence of permutation composition, see eq. (C.9). We take the $Y_{m}^{ \pm}$to be normalized, so that (by charge conservation) the non-vanishing two-point functions are

$$
\left\langle Y_{m}^{ \pm}\left(z_{1}, \bar{z}_{1}\right) Y_{m}^{\mp}\left(z_{2}, \bar{z}_{2}\right)\right\rangle=\left|z_{12}\right|^{-4 h_{m}^{Y}} .
$$


Inserting the OPE back into the four-point function, the leading short-distance coefficients $C_{\mathfrak{a}}$ give us information about the product of structure constants

$$
\begin{aligned}
& \left\langle R_{n}^{-}(\infty) O_{2}^{\text {(int) }}(1) Y_{n-1}^{+}(0)\right\rangle\left\langle R_{n}^{+}(\infty) O_{2}^{\text {(int) }}(1) Y_{n-1}^{-}(0)\right\rangle=\left|C_{1}\right|^{2}, \\
& \left\langle R_{n}^{-}(\infty) O_{2}^{\text {(int) }}(1) Y_{n+1}^{+}(0)\right\rangle\left\langle R_{n}^{+}(\infty) O_{2}^{\text {(int) }}(1) Y_{n+1}^{-}(0)\right\rangle=\left|C_{2}\right|^{2}
\end{aligned}
$$

(Recall that we must take $\left|G_{R}\left(x_{\mathfrak{a}}^{0}(u)\right)\right|^{2}$.) In the l.h.s. we actually have products of conjugate three-point functions/structure constants,

$$
C_{n, 2, m}^{R^{\mp} O^{(\mathrm{int})} Y^{ \pm}}=\left\langle R_{n}^{\mp} O_{2}^{(\mathrm{int})} Y_{m}^{ \pm}\right\rangle=C_{n, 2, m}^{R^{ \pm} O^{(\mathrm{int})} Y^{\mp}},
$$

(with the twists in the subscripts) and taking the explicit expressions for $C_{\mathfrak{a}}$, found from the expansions (5.18)-(5.19), we get

$$
\begin{aligned}
& \log C_{n, 2, n-1}^{R^{ \pm} O^{(\mathrm{int})} Y^{\mp}}=-\frac{n+2}{2} \log (n-1)+\frac{n^{2}-4 n-2}{2(n-1)} \log n, \\
& \log C_{n, 2, n+1}^{R^{ \pm} O^{(\text {int })} Y^{\mp}}=+\frac{n-2}{2} \log (n+1)-\frac{n^{2}+4 n-2}{2(n+1)} \log n .
\end{aligned}
$$

A similar situation takes place in the OPE $O_{2}^{(\text {int })} \sigma_{n}$, found in the limit $u \rightarrow 0$ of $G_{\sigma}(u, \bar{u})$. The two channels reveal twisted operators $\mathcal{Y}_{m}$ with zero R-charge and dimensions

$$
h_{m}^{\mathcal{Y}}=-\frac{5}{8}\left(\frac{m-2}{m}\right)+h_{m}^{\sigma}
$$

in a fusion rule

$$
\left[O_{[2]}^{(\mathrm{int})}\right] \times\left[\sigma_{[n]}\right]=\left[\mathcal{Y}_{[n-1]}\right]+\left[\mathcal{Y}_{[n+1]}\right]
$$

the terms in the r.h.s. corresponding to $x_{1}^{0}(u)$ and $x_{2}^{0}(u)$, respectively. The coefficients calculated from the expansion of $G_{\sigma}$ give structure constants as before:

$$
\begin{aligned}
& \log C_{n, 2, n-1}^{\sigma O^{(\mathrm{int})} \mathcal{Y}}=-\frac{(n+1)^{2}}{2 n} \log (n-1)+\frac{n^{2}-4 n-1}{2(n-1)} \log n \\
& \log C_{n, 2, n+1}^{\sigma O^{\text {(int) }} \mathcal{Y}}=+\frac{(n-1)^{2}}{2 n} \log (n+1)-\frac{n^{2}+4 n-1}{2(n+1)} \log n
\end{aligned}
$$

where $\left\langle\sigma_{n}(\infty) O_{2}^{(\mathrm{int})}(1) \mathcal{Y}_{m}(0)\right\rangle \equiv C_{n, 2, m}^{\sigma O^{(\mathrm{int})} \mathcal{Y}}$.

We have found that the operator algebras of Ramond fields with the deformation operator include non-BPS fields. These fields are consistent with the fractional spectral flow with $\xi=\frac{n}{n+1}$ of twisted non-BPS fields in the NS sector, recently found [61] to be a part of the OPEs of the deformation operator and NS chiral operators; see the discussion in section 6.5. A complete study of the algebras found here requires knowledge of OPEs such as $\left[Y_{n \pm 1}^{ \pm}\right] \times\left[O_{[2]}^{(\text {int })}\right]$. For that, the new fields have to be explicitly constructed. From our discussion of their properties, and in particular from the conformal dimension (5.20), we can infer that

$$
Y_{m}^{ \pm}(0)=G_{-\frac{1}{2 m}}^{ \pm} J_{-\frac{1}{m}}^{3} \sigma_{m}(0)
$$

This explicit construction should be sufficient for the study of the remaining OPEs by the computation of four-point functions such as $\left\langle Y_{[m]}^{-}(\infty) O_{[2]}^{\text {(int) }}(1) O_{[2]}^{(\text {int) }}(u, \bar{u}) Y_{[m]}^{+}\right\rangle$(which, incidentally, can be computed with the same covering map used here). 


\section{Analytic regularization and field renormalization}

We now turn to the calculation of the conformal dimension of Ramond fields in the interacting $\mathrm{SCFT}_{2}$. At second-order in perturbation theory, this requires computation of the integral (3.9), using the functions we have found in section 4 .

\subsection{Dotsenko-Fateev integrals}

We want to compute integrals $J=\int d^{2} u G(u, \bar{u})$, given an analytic expression for

$$
G(x)=C \frac{x^{\alpha_{1}}(x-1)^{\alpha_{2}}(x+n)^{\alpha_{3}}(x+n-1)^{\alpha_{4}}}{\left(x+\frac{n-1}{2}\right)^{\alpha_{5}}}, \quad \text { with } \quad\left\{\begin{array}{l}
\alpha_{1}+n-2=\alpha_{4}-n \\
\alpha_{2}-n-2=\alpha_{3}+n
\end{array}\right.
$$

from which $G(u, \bar{u})$ is obtained by inversion of the map (4.6). Both the Ramond function (4.35) and the bare twist function (4.38) have the form (6.1). We can perform a change of variables from $u$ to $x$ in the integral which, taking the special relation between the exponents into account, becomes

$$
J=\int d^{2} x\left|u^{\prime}(x) G(x)\right|^{2}=(4 n C)^{2} \int d^{2} x\left|\frac{[x(x+n-1)]^{\alpha_{4}-n}[(x-1)(x+n)]^{\alpha_{3}+n}}{\left(x+\frac{n-1}{2}\right)^{\alpha_{5}-2}}\right|^{2} .
$$

We then make the following change of variables [17],

$$
y(x)=-\frac{4(x-1)(x+n)}{(n+1)^{2}},
$$

such that every term in the new integrand is expressed simply in terms of $y$,

$$
\begin{aligned}
& J(n)=\frac{(4 n C)^{2}}{4}\left(\frac{n+1}{2}\right)^{4[a+b+c+1]} I(n), \\
& I(n) \equiv \int d^{2} y|y|^{2 a}|1-y|^{2 b}\left|y-w_{n}\right|^{2 c},
\end{aligned}
$$

where

$$
a=\alpha_{3}+n, \quad b=-\frac{\alpha_{5}-1}{2}, \quad c=\alpha_{4}-n, \quad w_{n}=\frac{4 n}{(n+1)^{2}} .
$$

We will refer to $I(n)$ as a 'Dotsenko-Fateev (DF) integral', as it has been studied in detail by Dotsenko and Fateev, as a representation of correlation functions in degenerate CFTs $[53-55] .{ }^{9}$

The properties of $I(n)$ crucially depend on the exponents of its critical points $y=$ $\left\{0, w_{n}, 1\right\}$. For example, the exponents for $G_{R}(x)$ are

$$
a_{R}=\frac{1}{2}+\frac{1}{4} n, \quad b_{R}=-\frac{3}{2}, \quad c_{R}=\frac{1}{2}-\frac{1}{4} n
$$

thus, for general $n$, all three critical points are branching points. The integral diverges at 1 and $\infty$, and vanishes at 0 for all $n$; at $w_{n}$, it converges for $n \leq 6$ and diverges for $n>6$. Clearly, some regularization procedure is needed. Following ref. [55], we now show that

\footnotetext{
${ }^{9}$ Cf. also refs. [56-58].
} 
$I(n)$ can be expressed in terms of hypergeometric functions, leading to a regularization by analytic continuation. We do this in two steps:

1. Assume that the parameters $a, b, c$ are such that the DF integral exists.

2. Express the integrals in terms of an analytic function of $a, b, c$ that is well-defined also for values of $a, b, c$, such as (6.7), for which the original integral diverges. (Such functions will turn out to be a product of hypergeometric and Gamma functions.) This leads to an extension of the definition of the integrals by their maximal analytic continuation.

As we shall see, the procedure is consistent.

Let us write $y \in \mathbb{C}$ as $y=y_{1}+i y_{2}$ in (6.5), and perform a rotation of $y_{2}$, such that $y_{2} \mapsto i(1-2 i \varepsilon) y_{2}$, with $\varepsilon$ a positive arbitrarily small parameter. Defining $v_{ \pm}=y_{1} \pm y_{2}$ (where $y_{2}$ now refers to the new, rotated coordinate), and expanding the integrand to first order in $\varepsilon$,

$$
\begin{aligned}
I=i \iint d y_{1} d y_{2}[ & \left.y_{1}^{2}-y_{2}^{2}(1-4 i \varepsilon)\right]^{a}\left[\left(y_{1}-1\right)^{2}-y_{2}^{2}(1-4 i \varepsilon)\right]^{b} \\
\times & {\left[\left(y_{1}-w_{n}\right)^{2}-y_{2}^{2}(1-4 i \varepsilon)\right]^{c} } \\
=\frac{i}{2} \iint d v_{-} d v_{+} & {\left[\left\{v_{-}-i \varepsilon\left(v_{-}-v_{+}\right)\right\}\left\{v_{+}+i \varepsilon\left(v_{-}-v_{+}\right)\right\}\right]^{a} } \\
\times & {\left[\left\{v_{-}-1-i \varepsilon\left(v_{-}-v_{+}\right)\right\}\left\{v_{+}-1+i \varepsilon\left(v_{-}-v_{+}\right)\right\}\right]^{b} } \\
& \times\left[\left\{v_{-}-w_{n}-i \varepsilon\left(v_{-}-v_{+}\right)\right\}\left\{v_{+}-w_{n}+i \varepsilon\left(v_{-}-v_{+}\right)\right\}\right]^{c} .
\end{aligned}
$$

The double integrals have been factorized into a product of two one-dimensional integrals,

$$
\begin{aligned}
I=\frac{i}{2} \int_{-\infty}^{+\infty} d v_{-} & {\left[v_{-}-i \varepsilon\left(v_{-}-v_{+}\right)\right]^{a}\left[v_{-}-1-i \varepsilon\left(v_{-}-v_{+}\right)\right]^{b} } \\
\times & \times\left[v_{-}-w_{n}-i \varepsilon\left(v_{-}-v_{+}\right)\right]^{c} \\
\times \int_{-\infty}^{+\infty} d v_{+} & {\left[v_{+}+i \varepsilon\left(v_{-}-v_{+}\right)\right]^{a}\left[v_{+}-1+i \varepsilon\left(v_{-}-v_{+}\right)\right]^{b} } \\
\times & \times\left[v_{+}-w_{n}+i \varepsilon\left(v_{-}-v_{+}\right)\right]^{c}
\end{aligned}
$$

because the variable $v_{ \pm}$only appears in the $v_{\mp}$ integral multiplied by the infinitesimal parameter $\varepsilon$. The effect of the $\varepsilon$-terms is to specify how the otherwise real integrals of

$$
f(\zeta)=\zeta^{a}(\zeta-1)^{b}\left(\zeta-w_{n}\right)^{c}, \quad \zeta \in \mathbb{C}, \quad w_{n} \in(0,1) \subset \mathbb{R},
$$

go around the points $0, w_{n}, 1$. To further disentangle the integrals, we split integration over $v_{+}$at $0, w_{n}, 1$, so that $\varepsilon$-terms can be ignored, while the $v_{-}$integrals go around the contours $\gamma_{k}$ dictated by the infinitesimal terms $\varepsilon\left(v_{-}-v_{+}\right)$as in figure 1(a),

$$
\begin{aligned}
I=\frac{i}{2}[ & \int_{-\infty}^{0} d v_{+} f\left(v_{+}\right) \int_{\gamma_{0}} d v_{-} f\left(v_{-}\right)+\int_{0}^{w_{n}} d v_{+} f\left(v_{+}\right) \int_{\gamma_{1}} d v_{-} f\left(v_{-}\right) \\
& \left.+\int_{w_{n}}^{1} d v_{+} f\left(v_{+}\right) \int_{\gamma_{2}} d v_{-} f\left(v_{-}\right)+\int_{1}^{\infty} d v_{+} f\left(v_{+}\right) \int_{\gamma_{3}} d v_{-} f\left(v_{-}\right)\right] .
\end{aligned}
$$




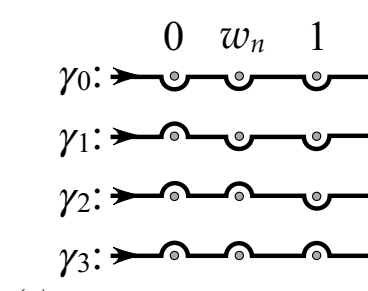

(a)

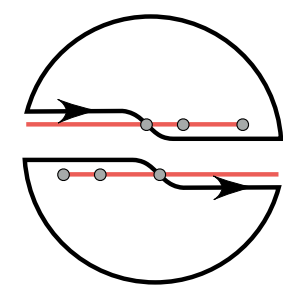

(b)

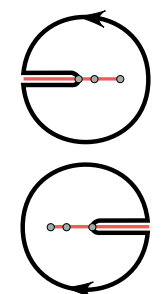

(c)

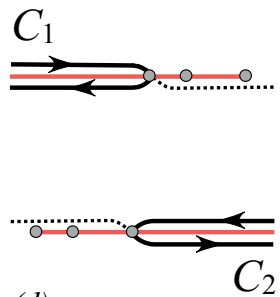

(d)

Figure 1. (a) Contours for the Dotsenko-Fateev integral; (b) Closing $\gamma_{1}$ and $\gamma_{2}$; (c) Deformation; (d) Final contours (ignoring circles at infinity). Red lines indicate branching cuts.

For example, for $v_{+} \in\left(0, w_{n}\right)$,

$$
\left.\varepsilon\left(v_{-}-v_{+}\right)\right|_{v_{-}=0}>0,\left.\quad \varepsilon\left(v_{-}-v_{+}\right)\right|_{v_{-}=w_{n}}<0,\left.\quad \varepsilon\left(v_{-}-v_{+}\right)\right|_{v_{-}=1}<0,
$$

hence the contour $\gamma_{1}$ goes above $v_{-}=0$, and below $v_{-}=w_{n}, 1$.

The function $f(\zeta)$ has branch cuts, so closing the contours $\gamma_{k}$ with semi-circles is nontrivial. Here is the point were our regularization procedure effectively starts. Assume that $a, b, c$ are such that the DF integral is convergent. Precisely, assume that

$$
a>-1, \quad b>-1, \quad c>-1, \quad 1+a+b+c<0,
$$

which ensures, respectively, convergence at the points $0,1, w_{n}, \infty$. Now close the contours $\gamma_{k}$ by making semicircles of radius $R \rightarrow \infty$ on the lower or upper plane. The curve $\gamma_{0}$ passes below every branch point, hence close the contour with a clockwise semicircle $\Gamma^{-}$on the lower plane; there are no poles inside $\gamma_{0} \cup \Gamma^{-}$, and $\lim _{R \rightarrow \infty} \int_{\Gamma^{-}} d v_{-} f\left(v_{-}\right) \rightarrow 0$ given our assumptions (6.11); hence $\int_{\gamma_{0}} d v_{-} f\left(v_{-}\right)=0$. Similarly, $\int_{\gamma_{3}} d v_{-} f\left(v_{-}\right)=0$, now with the contour on the upper plane. Thus only two terms remain in eq. (6.10).

If we try to close $\gamma_{1}$ or $\gamma_{2}$ in figure 1(a), we are deemed to cross branch cuts, and move to another Riemann sheet of $f\left(v_{-}\right)$. One way out of this is to cross the cut on a branching point, where $f\left(v_{-}\right)$is single-valued. That the integral exists at the branching points is assured by our assumptions (6.11). Thus we choose the branch cuts to align with the Real axis in two different ways: for the integral over $\gamma_{1}$ they extend to $-\infty$, and for $\gamma_{2}$ they extend to $+\infty$; then we close the contours with semi-circles as in figure 1(b). In one case, we cross the real axis at $v_{-}=0$, in the other at $v_{-}=w_{n}$. Next, we deform the contours as in figure 1(c). Given our assumptions (6.11), as $R \rightarrow \infty$ the integral over the (almost closed) circle vanishes, and we have $\int_{\gamma_{i}} d v_{-} f\left(v_{-}\right)=\int_{C_{i}} d v_{-} f\left(v_{-}\right)$for $i=1,2$, where the contours $C_{i}$ are shown in figure 1(d). Integration over $C_{i}$ is standard: the effect of coasting the two margins of a branch cut, turning at the branch point is to produce a phase $2 i \sin (\pi \theta)$.

Thus we arrive at the following form of (6.10),

$$
\begin{aligned}
I\left(a, b, c ; w_{n}\right)= & -s(a) \tilde{I}_{1}\left(a, b, c ; w_{n}\right) I_{2}\left(a, b, c ; w_{n}\right) \\
& -s(b) I_{1}\left(a, b, c ; w_{n}\right) \tilde{I}_{2}\left(a, b, c ; w_{n}\right),
\end{aligned}
$$


where $s(\theta) \equiv \sin (\pi \theta)$ and we have defined four 'canonical integrals':

$$
\begin{aligned}
& I_{1}\left(a, b, c ; w_{n}\right) \equiv \int_{1}^{\infty} d v_{+} v_{+}^{a}\left(v_{+}-1\right)^{b}\left(v_{+}-w_{n}\right)^{c} \\
& I_{2}\left(a, b, c ; w_{n}\right) \equiv \int_{0}^{w_{n}} d v_{-} v_{-}^{a}\left(1-v_{-}\right)^{b}\left(w_{n}-v_{-}\right)^{c} \\
& \tilde{I}_{1}\left(a, b, c ; w_{n}\right) \equiv \int_{-\infty}^{0} d v_{+}\left(-v_{+}\right)^{a}\left(1-v_{+}\right)^{b}\left(w_{n}-v_{+}\right)^{c} \\
& \tilde{I}_{2}\left(a, b, c ; w_{n}\right) \equiv \int_{w_{n}}^{1} d v_{-} v_{-}^{a}\left(1-v_{-}\right)^{b}\left(v_{-}-w_{n}\right)^{c}
\end{aligned}
$$

The $\tilde{I}_{1,2}$ can actually be written in terms of the $I_{1,2}$ with a different arrangement of their arguments:

$$
\tilde{I}_{1}\left(a, b, c ; w_{n}\right)=I_{1}\left(b, a ; c ; 1-w_{n}\right), \quad \tilde{I}_{2}\left(a, b, c ; w_{n}\right)=I_{2}\left(b, a ; c ; 1-w_{n}\right) .
$$

Also, by combining deformed contours such as the ones in figure 1, it can be shown [53] that $I_{1,2}\left(a, b, c, w_{n}\right)$ and $\tilde{I}_{1,2}\left(a, b, c, w_{n}\right)$, with the same arguments, form a linear system:

$$
s(b+c) I_{1}=s(a) \tilde{I}_{1}-s(c) \tilde{I}_{2}, \quad s(b+c) I_{2}=-s(a+b+c) \tilde{I}_{1}-s(b) \tilde{I}_{2} .
$$

The four canonical integrals are proportional to the Euler representation of the hypergeometric function $[66, \S 15.1]$,

$$
\begin{aligned}
& \int_{0}^{1} d t t^{\beta-1}(1-w t)^{-\alpha}(1-t)^{\gamma-\beta-1}=\frac{\Gamma(\beta) \Gamma(\gamma-\beta)}{\Gamma(\gamma)} F(\alpha, \beta ; \gamma ; w) \\
& \text { valid for }|\arg (1-w)|<\pi, \quad 0<\operatorname{Re}(\beta)<\operatorname{Re}(\gamma) .
\end{aligned}
$$

With the substitution $t=1 / v_{+}$in $I_{1}$, and $t=v_{-} / w_{n}$ in $I_{2}$, we find

$$
\begin{aligned}
& I_{1}\left(a, b ; c ; w_{n}\right)=\frac{\Gamma(-1-a-b-c) \Gamma(1+b)}{\Gamma(-a-c)} F\left(-c,-1-a-b-c ;-a-c ; w_{n}\right), \\
& I_{2}\left(a, b, c ; w_{n}\right)=\frac{\Gamma(1+a) \Gamma(1+c)}{\Gamma(2+a+c)} w_{n}^{1+a+c} F\left(-b, 1+a ; 2+a+c ; w_{n}\right) .
\end{aligned}
$$

The restrictions (6.16), required for both integrals to be represented by hypergeometrics, translate to $a, b, c$ as

$$
-1<a<1, \quad-1<b<-a, \quad-1<c<-1-a-b,
$$

and also $0<w_{n}<1$, cf. (6.6). These conditions are consistent with our starting hypothesis (6.11), therefore eq. (6.12) can be read as a product of hypergeometric and Gamma functions.

The 'canonical functions' (6.17) and (6.18) are analytic functions of each of the parameters $a, b, c$, on the domain of validity (6.19). This is evident for the Gamma functions, and is also true for the hypergeometrics, see [67, §2.1.6]. Note that in (6.17) and (6.18) what actually appears is the 'regularized hypergeometric function'

$$
\mathbf{F}(\alpha, \beta ; \gamma ; w) \equiv \frac{1}{\Gamma(\gamma)} F(\alpha, \beta ; \gamma ; w)
$$


which is an entire function of $\alpha, \beta, \gamma[67, \S 2.1 .6]$. In particular, $\mathbf{F}(\alpha, \beta ; \gamma ; w)$ is regular at $\gamma=-k$, with $k \in \mathbb{N}$, where the Gamma function develops a pole and $[66, \S 15.2]$

$$
\mathbf{F}(\alpha, \beta ;-k ; w)=\frac{\Gamma(\alpha+k+1) \Gamma(\beta+k+1)}{\Gamma(\alpha) \Gamma(\beta)(k+1) !} w^{k+1} F(\alpha+k+1, \beta+k+1 ; k+2 ; w) .
$$

Hence $I\left(a, b, c ; w_{n}\right)$ is analytic in $a, b, c$ separately. Consequently, an analytic continuation of $I\left(a, b, c ; w_{n}\right)$ to outside of the domain of definition (6.19) is unique, when it exists. We take this analytic continuation to be the definition of the DF integral (6.5) for arbitrary parameters. Note that it is not precluded that, outside the domain (6.19), I( $\left.a, b, c ; w_{n}\right)$ might develop a singularity - there may be a barrier to the analytic continuation - it just happens that, for the applications below, the continuation is, indeed, (almost) always well-defined.

\subsection{The integral for R-charged Ramond fields}

Let us apply our results to the Ramond function (4.35). As noted before, the parameters (6.7) do not lie within the domain (6.19), hence we are indeed using the analytic continuation. Eqs. (6.17), (6.18), (6.14) yield

$$
\begin{aligned}
& I_{1}\left(a_{R}, b_{R}, c_{R}\right)=\frac{\pi\left(4-n^{2}\right)}{32} w^{2} F\left(\frac{3}{2}, \frac{3}{2}+\frac{1}{4} n ; 3 ; w_{n}\right) \\
& I_{2}\left(a_{R}, b_{R}, c_{R}\right)=\frac{1}{s\left(\frac{1}{2}-\frac{n}{4}\right)} I_{1}\left(a_{R}, b_{R}, c_{R}\right) \\
& \tilde{I}_{1}\left(a_{R}, b_{R}, c_{R}\right)=-\frac{2 \sqrt{\pi} \Gamma\left(\frac{3}{2}+\frac{n}{4}\right)}{\Gamma\left(1+\frac{n}{4}\right)} F\left(-\frac{1}{2}+\frac{n}{4},-\frac{1}{2} ; 1+\frac{n}{4} ; 1-w_{n}\right) \\
& \tilde{I}_{2}\left(a_{R}, b_{R}, c_{R}\right)=-\frac{2 \sqrt{\pi} \Gamma\left(\frac{3}{2}-\frac{n}{4}\right)}{\Gamma\left(1-\frac{n}{4}\right)}(1-w)^{-n / 4} F\left(-\frac{1}{2}-\frac{n}{4},-\frac{1}{2} ; 1-\frac{n}{4} ; 1-w_{n}\right)
\end{aligned}
$$

Several observations are in order. The expression (6.22a) does not correspond immediately to the formula (6.17), because here we have $\Gamma(-1)$ in the denominator. In this case, we must use eq. (6.21) to find the correct expression for $I_{1}$ in (6.22a). Expression (6.22b) can be found immediately from (6.18). The factor $s(c)$ in $(6.22 \mathrm{~b})$ can be found either from $\Gamma(z) \Gamma(1-z)=\pi / \sin (\pi z)$, or from the linear system (6.15), by noting that in the present case we have

$$
s\left(a_{R}+c_{R}\right)=0, \quad s\left(a_{R}\right)=s\left(c_{R}\right), \quad s\left(a_{R}+b_{R}+c_{R}\right)=-s\left(b_{R}\right)=-1 .
$$

Eqs. (6.22c) and (6.24d) follow immediately from (6.14), but (6.24d) is only valid when $n$ is odd. For $n$ even there are two cases. When $n=4(k+1)$ a pole of the Gamma function in the denominator of $(6.24 \mathrm{~d})$ requires that we use eq. (6.21) again, leading to $\tilde{I}_{2}=\tilde{I}_{1}$. This can also be found from the linear system (6.15) by noting that, besides (6.23), now $s\left(b_{R}+c_{R}\right)=0$. 
All of the peculiarities above are taken into account if we simply replace the hypergeometrics by the well-behaved regularized hypergeometric,

$$
\begin{aligned}
& I_{1}\left(a_{R}, b_{R}, c_{R}\right)=\frac{\pi\left(4-n^{2}\right)}{32} w_{n}^{2} F\left(\frac{3}{2}, \frac{6+n}{4} ; 3 ; w_{n}\right) \\
& I_{2}\left(a_{R}, b_{R}, c_{R}\right)=\frac{1}{2} \Gamma\left(\frac{6-n}{4}\right) \Gamma\left(\frac{6+n}{4}\right) w_{n}^{2} F\left(\frac{3}{2}, \frac{6+n}{4} ; 3 ; w_{n}\right) \\
& \tilde{I}_{1}\left(a_{R}, b_{R}, c_{R}\right)=-2 \sqrt{\pi} \Gamma\left(\frac{6+n}{4}\right) \mathbf{F}\left(\frac{n-2}{4},-\frac{1}{2} ; \frac{n+4}{4} ; 1-w_{n}\right) \\
& \tilde{I}_{2}\left(a_{R}, b_{R}, c_{R}\right)=-\frac{2 \sqrt{\pi}}{\left(1-w_{n}\right)^{\frac{n}{4}}} \Gamma\left(\frac{6-n}{4}\right) \mathbf{F}\left(-\frac{n+2}{4},-\frac{1}{2} ; \frac{4-n}{4} ; 1-w_{n}\right)
\end{aligned}
$$

We can now use eqs. (6.12) and (6.4) to write

$$
J_{R}(n)=-\left(\frac{n+1}{32 n}\right)^{2}\left[\cos \left(\frac{n \pi}{4}\right) \tilde{I}_{1}(n) I_{2}(n)+I_{1}(n) \tilde{I}_{2}(n)\right], \quad\left\{\begin{array}{l}
n \neq 4 k+2 \\
k \in \mathbb{N}
\end{array}\right.
$$

Before we analyze this result further, let us consider what happens if $n=4 k+2$.

The case $\boldsymbol{n}=\boldsymbol{4} \boldsymbol{k}+\mathbf{2}$. When $n=4 k+2$, a pole of the Gamma function appears in $(6.22 \mathrm{~d})$ and $(6.24 \mathrm{~d})$, so $I(n)$ is infinite. We can isolate the divergence, however. First, we list again the four canonical integrals, now in terms of $k=\frac{n-2}{4}$,

$$
\begin{aligned}
& I_{1}(k)=-\frac{32 \pi k(k+1)(2 k+1)^{2}}{(4 k+3)^{4}} F\left(\frac{3}{2}, k+2 ; 3 ; \frac{8(2 k+1)}{(4 k+3)^{2}}\right) \\
& I_{2}(k)=\frac{32(2 k+1)^{2}}{(4 k+3)^{4}} \Gamma(1-k) \Gamma(k+2) F\left(\frac{3}{2}, k+2 ; 3 ; \frac{8(2 k+1)}{(4 k+3)^{2}}\right) \\
& \tilde{I}_{1}(k)=-2 \sqrt{\pi} \Gamma(k+2) \mathbf{F}\left(-\frac{1}{2}, k ; k+\frac{3}{2} ; \frac{(4 k+1)^{2}}{(4 k+3)^{2}}\right) \\
& \tilde{I}_{2}(k)=-\frac{2 \sqrt{\pi}(4 k+3)^{2 k+1}}{(4 k+1)^{2 k+1}} \Gamma(1-k) \mathbf{F}\left(-\frac{1}{2},-k-1 ; \frac{1}{2}-k ; \frac{(4 k+1)^{2}}{(4 k+3)^{2}}\right)
\end{aligned}
$$

Here we note that in this case we have $s\left(a_{R}\right)=s\left(c_{R}\right)=0$ besides (6.23), and the linear system (6.15) is not valid anymore. This is related to the fact that there is now only one branch point in the canonical integrals, instead of the three branchings of the general case. Eq. (6.12) is, however, still valid. Moreover, we have $I_{2}(k)=-I_{1}(k) / \sin (\pi k)$. The sine is cancelled in eq. (6.12),

$$
I(k)=-I_{1}(k)\left(\tilde{I}_{1}(k)+\tilde{I}_{2}(k)\right) .
$$

Now the only singularity comes from the pole of $\Gamma(1-k)$ in (6.26d). Making $k \rightarrow k+\epsilon$, we have (cf. [67], §1.17 eq. (11))

$$
\Gamma(1-k-\epsilon)=\frac{(-1)^{k-1}}{(k-1) !}\left[-\frac{1}{\epsilon}+\psi(k)+\mathrm{O}(\epsilon)\right]
$$




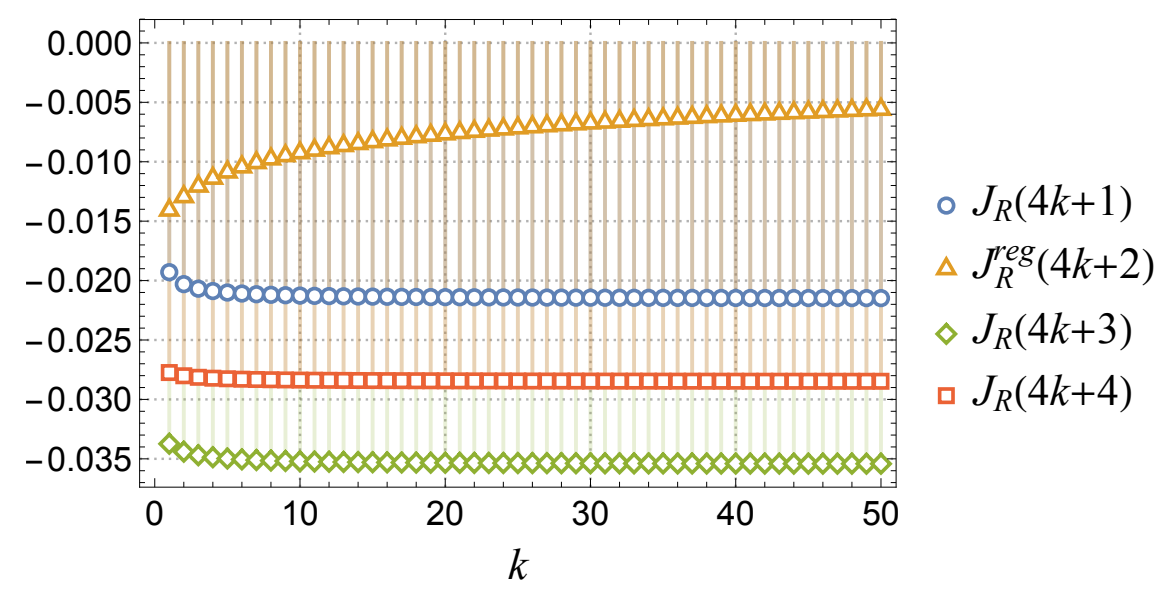

Figure 2. $J_{R}(n)$ (and its regularization) for charged Ramond fields.

from which we separate the finite part and the divergence:

$$
\begin{aligned}
\tilde{I}_{2}(k) & \equiv \frac{1}{\epsilon} \tilde{I}_{2}^{\text {sing }}(k)+\tilde{I}_{2}^{r e g}(k) \\
\tilde{I}_{2}^{\text {sing }}(k) & =\frac{(-1)^{k} 2 \sqrt{\pi}(4 k+3)^{2 k+1}}{(4 k+1)^{2 k+1}(k-1) !} \mathbf{F}\left(-\frac{1}{2},-k-1 ; \frac{1}{2}-k ; \frac{(4 k+1)^{2}}{(4 k+3)^{2}}\right) \\
\tilde{I}_{2}^{r e g}(k) & =\frac{(-1)^{k-1} 2 \sqrt{\pi}(4 k+3)^{2 k+1} \psi(k)}{(4 k+1)^{2 k+1}(k-1) !} \mathbf{F}\left(-\frac{1}{2},-k-1 ; \frac{1}{2}-k ; \frac{(4 k+1)^{2}}{(4 k+3)^{2}}\right)
\end{aligned}
$$

where $\psi(\zeta)$ is the digamma function. Using (6.27) and (6.4), we end up with

$$
\begin{aligned}
J_{R}(k) & =J_{R}^{\text {reg }}(k)+\epsilon^{-1} J_{R}^{\text {sing }}(k) \\
J_{R}^{r e g}(k) & =-\left(\frac{3+4 k}{128(1+2 k)}\right)^{2} I_{1}(k)\left[\tilde{I}_{1}(k)+\tilde{I}_{2}^{r e g}(k)\right], \\
J_{R}^{\text {sing }}(k) & =-\left(\frac{3+4 k}{128(1+2 k)}\right)^{2} I_{1}(k) \tilde{I}_{2}^{\text {sing }}(k) .
\end{aligned}
$$

which is the final regularized expression for $J_{R}$ when $n=4 k+2$.

Comments. We present a unified plot of $J_{R}(n)$ for every $n$ in figure 2 ; for $n=4 k+2$, we plot the regularized function $J_{R}^{r e g}(k)$. One can distinguish a peculiar "almost periodicity" of the function, with period 4 . We believe that this might be related to some combinatoric relation between the twists of $R_{n}^{ \pm}$and the twists of the interaction operators appearing in the four-point function. As can be seen from figure $2, J_{R}(n)$ stabilizes around small, negative values for large $n$. As a reference, for $k=30$ we have

$$
\begin{aligned}
& J_{R}(4 k+1) \approx-0.0215279 \quad J_{R}^{r e g}(4 k+2) \approx-0.0036010 \\
& J_{R}(4 k+3) \approx-0.0354618 \quad J_{R}(4 k+4) \approx-0.0284950
\end{aligned}
$$

Note that an analytic form of $J_{R}(n)$ for large $n$ is very hard to find because it involves taking simultaneous limits of the multiple arguments of the hypergeometric function. 


\subsection{The integral for bare twists}

The function $G_{\sigma}(x)$ also has the form (6.1), and

$$
J_{\sigma}(n)=\int d^{2} x\left|u^{\prime}(x) G_{\sigma}(x)\right|^{2}=\left[\frac{1}{2} n(n+1) C_{\sigma}\right]^{2} I(n),
$$

where $I(n)$ is a DF integral (6.5) with exponents

$$
a_{\sigma}=\frac{(n+1)^{2}}{4 n}, \quad b_{\sigma}=-\frac{3}{2}, \quad c_{\sigma}=-\frac{(n-1)^{2}}{4 n} .
$$

The canonical integrals

$$
\begin{aligned}
& I_{1}\left(a_{\sigma}, b_{\sigma}, c_{\sigma}\right)=-\frac{\pi(n-1)^{2}}{2(n+1)^{2}} F\left(\frac{3}{2}, \frac{1+6 n+n^{2}}{4 n} ; 3 ; w_{n}\right) \\
& I_{2}\left(a_{\sigma}, b_{\sigma}, c_{\sigma}\right)=\frac{8 n^{2}}{(n+1)^{4}} \Gamma\left(1-\frac{(n-1)^{2}}{4 n}\right) \Gamma\left(1+\frac{(n+1)^{2}}{4 n}\right) F\left(\frac{3}{2}, \frac{1+6 n+n^{2}}{4 n} ; 3 ; w_{n}\right) \\
& \tilde{I}_{1}\left(a_{\sigma}, b_{\sigma}, c_{\sigma}\right)=-2 \sqrt{\pi} \Gamma\left(1+\frac{(n+1)^{2}}{4 n}\right) \mathbf{F}\left(-\frac{1}{2}, \frac{(n-1)^{2}}{4 n} ; \frac{1+4 n+n^{2}}{4 n} ; 1-w_{n}\right) \\
& \tilde{I}_{2}\left(a_{\sigma}, b_{\sigma}, c_{\sigma}\right)=-2 \sqrt{\pi} \Gamma\left(1-\frac{(n-1)^{2}}{4 n}\right)\left(1-w_{n}\right)^{-\frac{1+n^{2}}{4 n}} \\
& \mathbf{F}\left(-\frac{1}{2},-\frac{(1+n)^{2}}{4 n} ;-\frac{1-4 n+n^{2}}{4 n} ; 1-w_{n}\right)
\end{aligned}
$$

are all well-defined and convergent for all values of $n \in \mathbb{N}$ - the arguments of the Gamma functions are never a negative integer (for $n>1$ ).

We plot the values of

$$
J_{\sigma}(n)=-\left(\frac{n+1}{32 n}\right)^{2}\left[s(a) \tilde{I}_{1}(n) I_{2}(n)+s(b) I_{1}(n) \tilde{I}_{2}(n)\right]
$$

in figure 3. We can see again an approximate periodicity, with period 4 , similar to what happens in the Ramond case. We can give the following numerical values for $k=30$ :

$$
\begin{array}{ll}
J_{\sigma}(4 k+1) \approx-0.0214398 & J_{\sigma}(4 k+2) \approx-1.11106 \\
J_{\sigma}(4 k+3) \approx-0.035381 & J_{\sigma}(4 k+4) \approx-0.028456
\end{array}
$$

to be compared with the corresponding values for $J_{R}(n)$ given above. For $n=4 k+2$, $J_{\sigma}(n)$ grows with $n$, instead of stabilizing around a small value; note that these values of $n$ are also those for which the Ramond integral $J_{R}(n)$ diverged, and had to be regularized.

\subsection{Renormalization of Ramond and twist fields}

The renormalized dimension of the Ramond operators is given by eq. (3.10). To first order in $\lambda$, it would be proportional do the structure constant of the three-point function

$$
\left\langle R_{[n]}^{-}(\infty) O_{[2]}^{(\mathrm{int})}(z) R_{[n]}^{+}(0)\right\rangle=0,
$$




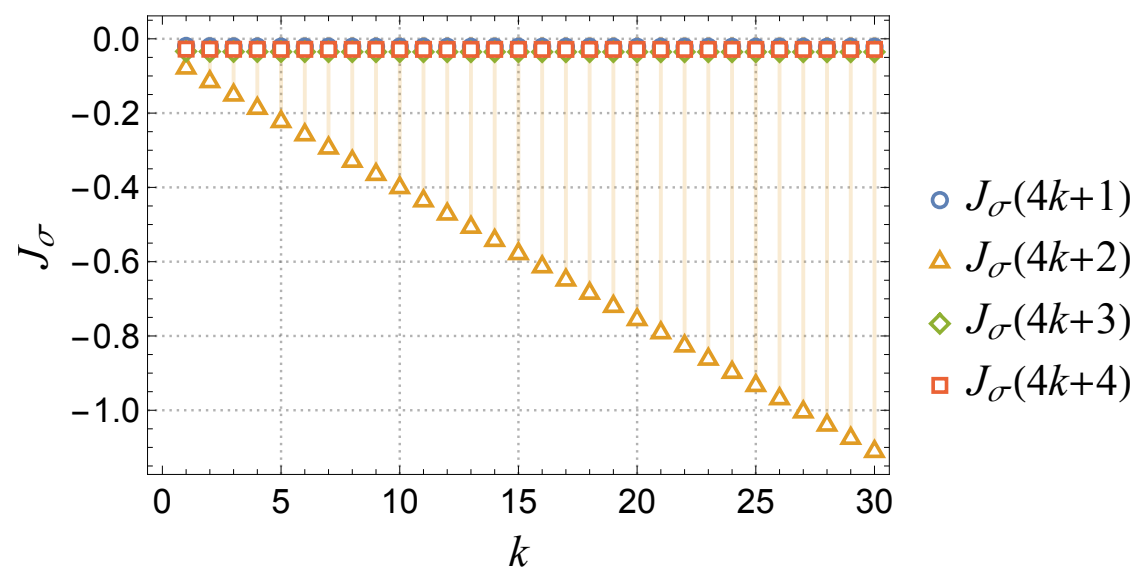

Figure 3. Numerical result of the integral (6.35) for twist fields.

which vanishes at the free orbifold point because $R_{[n]}^{-}$does not appear in the OPE $O_{[2]}^{(\text {int })}(z, \bar{z}) R_{[n]}^{+}(0)$. We are thus left with a correction only at order $\lambda^{2}$,

$$
\Delta_{n}^{R}(\lambda)=\frac{n}{2}+\frac{\pi}{2} \lambda^{2}\left|J_{R}(n)\right|+\cdots,
$$

where $\frac{1}{2} n=h_{n}^{R}(0)+\tilde{h}_{n}^{R}(0)=\Delta_{n}^{R}(0)$ is the (total) dimension of $R_{[n]}^{ \pm}(z, \bar{z})$ in the free theory. Here it should be understood that $J_{R}(4 k+2) \equiv J_{R}^{r e g}(k)$. The renormalized fields are therefore given by

$$
R_{[n]}^{ \pm(r e n)}(z, \bar{z})=\Lambda^{\frac{\pi}{2} \lambda^{2} J_{R}(n)} R_{[n]}^{ \pm}(z, \bar{z}),
$$

where $\Lambda \ll 1$ is the cutoff appearing in (3.4). We can also give the renormalization of the structure constant; from eq. (3.11),

$$
\left\langle R_{n}^{-}(\infty) O_{[2]}^{(\mathrm{int})}(1) R_{n}^{+}(0)\right\rangle=\lambda J_{R}(n)+\cdots
$$

We thus have concluded that for generic $n<N$ the dimension of the Ramond field slightly increases in the perturbed theory. The case $n=2$, which is the smallest value of $n$ for a twisted field, ${ }^{10}$ is special. The function (4.35) loses the singularity at $x=0$. It is interesting to see how this arises as a consequence of the permutation structure of the twists: we have seen that the OPE channel with $x \rightarrow 0$ results in a field with twist $n-1$; in this case that would be an untwisted field. As a consequence of eq. (4.18), this would mean that the 2-cycles of $O_{2}^{(\text {int) }}$ and $R_{2}^{ \pm}$entering the correlators in this channel are all the same; but such a correlator would involve only $\mathbf{s}=2=n$ copies instead of $n+1$, and therefore require a genus-one covering surface. Most importantly, the $n=2$ twisted Ramond fields do not renormalize - it can be easily checked that

$$
J_{R}(2)=0 .
$$

\footnotetext{
${ }^{10}$ We note that the results above do not apply directly to the untwisted Ramond field $(n=1)$. In particular, the DF integral has a different structure, with one less critical point.
} 
For that, it suffices to look at eqs. (6.25) and (6.24), since $\cos \left(\frac{\pi}{2}\right)=0$ and $I_{1}(2)=0$ while the other canonical functions are finite.

The same analysis holds for the bare twists: their dimension in the perturbed theory becomes

$$
\Delta_{n}^{\sigma}(\lambda)=\frac{1}{2}\left(n-\frac{1}{n}\right)+\frac{\pi}{2} \lambda^{2}\left|J_{\sigma}(n)\right|+\cdots,
$$

and the renormalized twist operators are

$$
\sigma_{[n]}^{(\mathrm{ren})}(z, \bar{z})=\Lambda^{\frac{\pi}{2} \lambda^{2} J_{\sigma}(n)} \sigma_{[n]}(z, \bar{z}) .
$$

The structure constant

$$
\left\langle\sigma_{[n]}(\infty) O_{[2]}^{(\mathrm{int})}(1) \sigma_{[n]}(0)\right\rangle=\lambda J_{\sigma}(n)+\cdots
$$

which also vanishes in the free theory, acquires a non-vanishing value at first-order.

The regularization of the divergent integral $J$ described in this section gives welldefined, finite two-point functions in the deformed theory, to second order in $\lambda$. Here we have considered the renormalization of bare twists and Ramond fields, but the method is more general, and can be applied to all sectors of the $\mathrm{SCFT}_{2}$. Our procedure relied on the fact that $J$ can be reduced to a Dotsenko-Fateev integral for the functions $G_{R}(x)$ and $G_{\sigma}(x)$. This, in turn, relied on the structure of these functions, which had the form (6.1). It is not hard to check that, for any primary twisted $\mathscr{O}_{[n]}$ that we insert in the general correlation function (4.1), the corresponding $G(x)$ always has the form (6.1), including the specific relations between the pairs of exponents $\alpha_{1}, \alpha_{3}$ and $\alpha_{2}, \alpha_{4}$.

To see that this is true, one can reverse-engineer the reasoning developed in section 5 . Given an operator $\mathscr{O}_{n}$ consider the correlator

$$
G(u, \bar{u})=\left\langle\mathscr{O}_{[n]}^{\dagger}(\infty) O_{[2]}^{(\mathrm{int})}(1) O_{[2]}^{(\mathrm{int})}(u, \bar{u}) \mathscr{O}_{[n]}(0)\right\rangle .
$$

This function must be singular in the short-distance limit $u \rightarrow 0$, and consistent with the OPE rule (5.1). The associated function $G(x)$ must therefore be singular when $x$ goes to one of the values $x_{\mathfrak{a}}^{0}$ for $u \rightarrow 0$, or $x_{\mathfrak{a}}^{\infty}$ for $u \rightarrow \infty$, where $x_{\mathfrak{a}}^{0}, x_{\mathfrak{a}}^{\infty}$ are the channels in the limits $u \rightarrow 0$ and $u \rightarrow \infty$, respectively; see appendix B. This fixes the numerator of

$$
G(x)=C \frac{\left(x-x_{1}^{0}\right)^{\alpha_{1}}\left(x-x_{2}^{\infty}\right)^{\alpha_{2}}\left(x-x_{2}^{0}\right)^{\alpha_{3}}\left(x-x_{1}^{\infty}\right)^{\alpha_{4}}}{\left(x-x_{2}^{1}\right)^{\alpha_{5}}}
$$

while the denominator is fixed similarly by the channels in $u \rightarrow 1$. But (6.47) is just another way to write (6.1). Note that this argument only makes use of the properties of the function $u(x)$ and its inverses, i.e. only on the structure of the twists in the correlator - not on the specifics of $\mathscr{O}_{n}$ nor, even, on the properties of $O_{2}^{(\text {int })}$. Thus $\mathscr{O}_{[n]}$ can be, say, a primary NS field, an R-charged or R-neutral Ramond ground state, or a bare twist field; also, we can replace $O_{[2]}^{(\text {int })}$ by, say, the simplest chiral NS primaries $O_{[2]}^{(p, q)}$ (defined e.g. in $[6])$.

Having proved that $G(x)$ must have the structure (6.47), it remains for us to show that the exponents satisfy the two relations in (6.1). This is also a consequence of the OPEs. 


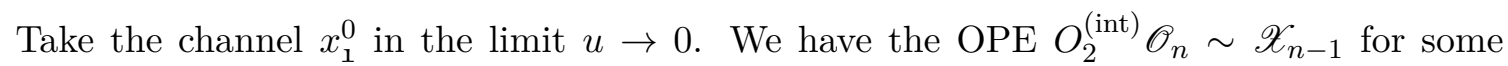
operator of twist $n-1$, whose dimension is fixed by the power of $u$ appearing in $G\left(x_{1}^{0}(u)\right)$. Since $G\left(x_{1}^{0}(u)\right) \sim\left[x_{1}^{0}(u)\right]^{\alpha_{1}}$, using (B.1) we have $G\left(x_{1}^{0}(u)\right) \sim u^{\frac{\alpha_{1}}{n-1}}$, hence the holomorphic dimension of $\mathscr{X}_{n-1}$ is

$$
h_{n-1}^{\mathscr{X}}=\frac{\alpha_{1}}{n-1}+h_{n}^{\mathscr{O}}+h_{2}^{O^{(\mathrm{int})}} .
$$

Now, in the limit $u \rightarrow \infty$, we will have the OPE $O_{2}^{\text {(int) }} \mathscr{O}_{n}^{\dagger} \sim \mathscr{X}_{n-1}^{\dagger}$. Using (B.2), we now have $G\left(x_{1}^{\infty}(u)\right) \sim u^{\frac{\alpha_{4}}{n-1}}$, and the dimension of $\mathscr{X}_{n-1}^{\dagger}$ is

$$
h_{n-1}^{\mathscr{X}^{\dagger}}=\frac{\alpha_{4}}{n-1}+h_{n}^{\mathscr{O}}-h_{2}^{O^{(\mathrm{int})}}
$$

with a minus sign in front of $h_{2}^{O^{(\text {int })}}$ because we must conjugate $O_{2}^{(\text {int })}$ to $\infty$. But $\mathscr{X}_{m}$ and $\mathscr{X}_{m}^{\dagger}$ have the same dimension, so subtracting eqs. (6.48) and (6.49) we find

$$
\frac{\alpha_{1}-\alpha_{4}}{n-1}=2 h_{2}^{O(\mathrm{int})}
$$

which, since $h_{2}^{O^{(\mathrm{int})}}=1$, gives the first relation in (6.1). The second relation, between $\alpha_{1}$ and $\alpha_{3}$, is found similarly in the channels $x_{2}^{0}$ and $x_{2}^{\infty}$, completing the proof that (6.1) holds in general.

Thus we have shown that, for any primary twisted filed $\mathscr{O}_{[n]}$, we can always reduce $J_{\mathscr{O}}$ to a Dotsenko-Fateev integral, for some set of parameters $a, b, c$. Then, we can apply our regularization procedure and subsequent renormalization of the two-point function $\left\langle\mathscr{O}_{[n]}^{\dagger} \mathscr{O}_{[n]}\right\rangle-i f$ that is necessary. A very important example of fields for which there is no renormalization is the class of BPS-protected NS chiral twisted fields. Explicit computation of their non-renormalization was given in [17], for $O_{n}^{(0,0)}$, the lowest-weight operator in the $n$-twisted sector of the NS chiral ring [68], with $h_{n}^{\mathrm{NS}}=\frac{n-1}{2}=j^{3}$. (The descendants of $\mathrm{O}_{2}^{(0,0)}$ give the deformation operator $O_{2}^{(\mathrm{int})}$.) The four-point function $G_{O}(x)$ was found by the same method of section 4, see eq. (D.6) of ref. [17]. It has the form (6.1), and gives rise to a Dotsenko-Fateev integral with exponents $a_{O}=1, b_{O}=-\frac{3}{2}, c_{O}=0$. Since $c_{O}=0$, the integral (6.5) simplifies, and can be computed directly in terms of Gamma functions, as done in appendix D of ref. [17], without the need to resorting to the hypergeometric regularization machinery. Nevertheless, it is interesting to confirm that our formulae do give the same result, i.e. $J_{O}=0$. Inserting $a_{O}, b_{O}$ and $c_{O}$ into our canonical functions (6.17)(6.18), we find

$$
I_{1}=0, \quad I_{2}=-4-\frac{2\left(w_{n}-2\right)}{\sqrt{1-w_{n}}}, \quad \tilde{I}_{1}=-4, \quad \tilde{I}_{2}=\frac{2\left(w_{n}-2\right)}{\sqrt{1-w_{n}}}
$$

and $s\left(a_{O}\right)=0$, so

$$
J_{O}(n)=-\left[\frac{1}{2} n(n+1) C_{O}\right]^{2}\left(s\left(a_{O}\right) \tilde{I}_{1}(n) I_{2}(n)+s\left(b_{O}\right) I_{1}(n) \tilde{I}_{2}(n)\right)=0
$$

as expected. 
Let us point out that our regularization and renormalization procedure is even more general. It can be extended almost intactly for the analysis of two-point functions of operators with a more complicated twist structure. In ref. [59] we have studied the doublecycle composite Ramond fields $R_{[n]}^{ \pm} R_{[m]}^{ \pm}(z, \bar{z})$. In this case, the covering map is more complicated, and, correspondingly, so is the form of $G(x)$ which generalizes (6.1); but just as explained above, there are relations between exponents which allow a transformation of $J(n, m)$ into a Dotsenko-Fateev integral, and then everything follows as in here.

\subsection{On spectral flow}

The spectral flow automorphism of the $\mathcal{N}=4$ super-algebra [69] acts on the Virasoro and R-current modes as

$$
\begin{aligned}
L_{\ell} \mapsto L_{\ell}^{\prime} & =L_{\ell}-J_{\ell}^{3} \xi+\frac{c}{24} \xi^{2} \delta_{\ell, 0} \\
J_{\ell}^{3} \mapsto J_{\ell}^{\prime 3} & =J_{\ell}^{3}-\frac{c}{12} \xi \delta_{\ell, 0}
\end{aligned}
$$

where $\xi$ is the spectral flow parameter. Hence an operator with conformal weight $h$ and R-charge $j^{3}$ is mapped to an operator with

$$
h^{\prime}=h-j^{3} \xi+\frac{c}{24} \xi^{2}, \quad j^{\prime 3}=j^{3}-\frac{c}{12} \xi,
$$

and NS (anti-)chiral fields flow to Ramond ground states:

$$
h^{\mathrm{NS}}= \pm j^{3} \quad \mapsto \quad h^{\prime}=\frac{1}{24} c \quad \text { for } \quad \xi= \pm 1 .
$$

Our renormalized fields $R_{[n]}^{ \pm}$, which are Ramond ground states of the $n$-wound string, have conformal weight

$$
h_{n}^{\mathrm{R}}=\frac{1}{4} n<\frac{1}{4} N,
$$

the bound $n<N$ being due to our calculation at order $N^{-1}$; the field with $n=N$ scales as $N^{-2}$ for large $N$, and requires a genus-one covering map. In the free theory, in the presence of a $n$-twist, it is possible to consider the $Z_{n}$ orbifold $\mathcal{N}=4$ SCFT with $c=6 n$, whose conserved currents are defined by adding the $n$ copies entering the twist. For example, taking the cycle to be $(1, \cdots, n)$, the $n$-twisted CFT has stress tensor and Virasoro modes ${ }^{11}$

$$
T(z)=\sum_{I=1}^{n} T_{I}(z), \quad L_{k} \sigma_{(1, \cdots, n)}(0)=\oint \frac{d z}{2 \pi i} z^{1+k} \sum_{I=1}^{n} T_{I}(z) \sigma_{(1, \cdots, n)}(0) .
$$

The modes $L_{k}$, with $k \in \mathbb{Z}$, are well defined: the twist shuffles the terms in the summation over $I$, but the summation itself is preserved. Spectral flow of this $n$-twisted algebra by $\xi=1$, when applied to the NS chiral field $O_{(1, \cdots, n)}^{(0,0)}$ with $h_{n}^{\mathrm{NS}}=\frac{n-1}{2}=j_{n}^{3}$, gives the Ramond field $R_{(1, \cdots, n)}^{-}$, with $h^{\prime}=\frac{1}{4} n$ and $j^{\prime 3}=-\frac{1}{2}$. Starting with the anti-chiral NS and flowing by $\xi=-1$, we get $R_{(1, \cdots, n)}^{+}$, etc.

\footnotetext{
${ }^{11}$ In the presence of $\sigma_{(n)}$ we can also define the usual fractional modes $L_{\frac{k}{n}}, J_{\frac{k}{n}}^{3}$, etc., as well as a 'fractional spectral flow' which is an automorphism of the fractional algebra [61].
} 
As shown by eq. (6.50), the dimension of the field $O_{(1, \cdots, n)}^{(0,0)}$ is protected in the deformed theory, although $R_{(1, \cdots, n)}^{ \pm}$is renormalized. How is this to be reconciled with the spectral flow between them? The answer is that the symmetry algebra of the $n$-twisted CFT, i.e. the $\mathcal{N}=(4,4)$ SCFT with central charge $c=6 n<6 N$, is not preserved after the deformation by the interaction $O_{[2]}^{(\mathrm{int})}$. The basic reason for this is that the twist can join two strings into a longer string.

Let us discuss this in more detail. The deformed SCFT has deformed charges $T^{(\lambda)}(z)$, $J^{(\lambda) a}(z), G^{(\lambda) \alpha A}(z)$, which must close under an operator algebra. If this algebra has an automorphism, then we can define a spectral flow between the deformed states. The deformed charges are difficult to describe explicitly. In particular, obtaining the stresstensor $T^{(\lambda)}(z)$ is subtle, as explained in [39], since one cannot naïvely make a variation of the action (3.1). Instead, one can use the prescription of Sen [70] to obtain the Virasoro modes by the following action on a field $\Phi$,

$$
L_{k}^{(\lambda)} \Phi(0)=\left(L_{k}+\lambda \delta L_{k}\right) \Phi_{0}=L_{k} \Phi(0)+\lambda \oint_{|z|=\epsilon} d \bar{z} z^{k+1} O_{[2]}^{(\mathrm{int})}(z, \bar{z}) \Phi(0) .
$$

See also [39], and [71] for a discussion of this formula. As shown in [70], the modes $L_{k}^{(\lambda)}$ satisfy the Virasoro algebra with the same central charge as the unperturbed algebra of the $L_{k}$, which are derived from the unperturbed tensor $T(z)$. Such preservation of the Virasoro algebra after the deformation (6.56) requires computing $\left[L_{k}, \delta L_{l}\right]$, which in turn includes computing the integral

$$
\underset{z \text { around } w}{\quad} d z z^{l+1} T(z) O_{[2]}^{(\mathrm{int})}(w, \bar{w}) .
$$

Now, $O_{[2]}^{(\text {int })}$ is made out of the sum over the conjugacy class of $Z_{2}$ cycles in $S_{N}$, hence it involves all the $N$ copies of the orbifold. Therefore the integral above is only defined if $T(z)$ also includes all the $N$ copies of the fields. For example, if we take the $n$-twisted algebra made by the modes (6.55), when going around the twist $\sigma_{(n, n+1)}$ the integral would not be defined. In other words, the Virasoro algebra with $c=6 n$ is inconsistent with the deformation (3.1) for $n<N$.

Let us also stress another important detail of our computation of the corrected dimension: since we perform the integral $J_{R}$ by changing coordinates to the covering surface, we automatically include all the conjugacy classes of the permutations inside the four-point function, which are taken into account by the very nature of the covering map, as we have extensively discussed in section 5. Thus we are truly computing the renormalized fields in the theory deformed by $O_{[2]}^{\text {(int) }}$, rather than by a non- $S_{N}$-invariant operator such as, say, $O_{(1,2)}^{(\text {int })}$.

Thus spectral flow between $R_{[n]}^{ \pm}$and $O_{[n]}^{(0,0)}$ is not preserved after the deformation by $O_{[2]}^{(\text {int })}$, unless $n=N$. Spectral flow of the full orbifold theory, with $c=6 N$ is, however, (expected to be) preserved. But, in the full orbifold theory, $R_{[n]}^{ \pm}$is not a "true" Ramond ground state - it is a mix of a $n$-twisted string in a Ramond ground state, with $N-n$ untwisted strings in the NS ground state. Thus one would not expect $R_{[n]}^{ \pm}$to be BPS 
protected. Explicitly, taking $\xi=1$ and with $c=6 N$, the field $R_{[n]}^{-}$with $h_{n}^{\mathrm{R}}=\frac{1}{4} n$ and $j^{3}=-\frac{1}{2}$ flows to a state with

$$
h^{\prime}=\frac{n+N-2}{4}, \quad j^{\prime 3}=\frac{N-1}{2}
$$

which is only chiral for $n=N$. Conversely, the NS chiral $O_{[n]}^{(0,0)}$ flows to a state with

$$
h^{\prime}=\frac{1}{4} N, \quad j^{\prime 3}=-\frac{N-n+1}{2}
$$

which is a "true" Ramond ground state made by composing twisted and untwisted Rcharged and R-neutral Ramond fields; when $n=N$, this field is simply $R_{[N]}^{-}$.

Note that this latter state with twist $N$ is indeed protected, as far as our computation is concerned, since, as shown in eq. (4.22), at leading order in $1 / N$, the four-point function involving the $R_{[N]}^{ \pm}$vanishes, and there is no correction to their dimensions. For the singlecycle fields considered here, this protection is rather trivial, being due simply to the large$N$ approximation. But we have shown in [59] that the protection is again observed in composite fields $R_{\left[m_{1}\right]}^{\alpha} R_{\left[m_{2}\right]}^{\beta}(z, \bar{z})$ with dimension $h^{\mathrm{R}}=\frac{1}{4} N$, for which $m_{1}+m_{2}=N$. In this case, there is a genus-zero contribution to the four-point function, and protection comes from a non-trivial DF integral being zero. Because of spectral flow, we expect that such results generalize for any composite Ramond field whenever the sum of the composing twists add to $N$.

\section{Discussion}

The investigation of the twisted Ramond sector of marginally-deformed D1-D5 $\mathrm{SCFT}_{2}$ presented in this paper is based on the explicit construction of the large- $N$ limit of the fourpoint function (4.24) of two R-charged Ramond fields and two scalar modulus operators $O_{[2]}^{(\text {int })}(z, \bar{z})=\epsilon_{A B} G_{-\frac{1}{2}}^{-A} \tilde{G}_{-\frac{1}{2}}^{-\dot{B}} O_{[2]}^{(0,0)}(z, \bar{z})$. We have found that $R_{[n]}^{\alpha}$ undergoes renormalization for all twists $2<n<N$, and is protected for the maximal and minimal twist values, $n=N$ and $n=2$. The fields $\sigma_{[n]}$ also renormalize for $n<N$. In fact, these four-point functions provide dynamical information about both theories: the "free-orbifold point" $\mathrm{SCFT}_{2}$, and its marginal deformation, at second order in $\lambda$. In what follows, we will briefly address a some open problems whose solutions can eventually be reached by adapting the methods developed in the present paper.

More on the properties of non-BPS fields. The four-point functions that we have calculated can be used not only for accessing the deformed $\mathrm{SCFT}_{2}$, but also to give a more complete description of the free orbifold itself. For example, the OPE data we have extracted from short-distance limits reveal important features of the Ramond sector of the free $\mathrm{SCFT}_{2}$, such as the conformal weights, R-charges and a few structure constants of the non-BPS twisted Ramond operators $Y_{n \pm 1}^{ \pm}$given by eq. (5.31). Their four-point functions with the deformation operator,

$$
\left\langle Y_{[m]}^{-}(\infty) O_{[2]}^{(\mathrm{int})}(1) O_{[2]}^{(\mathrm{int})}(u, \bar{u}) Y_{[m]}^{+}(0)\right\rangle,
$$


can be explicitly constructed by the same covering map and the same methods used here. Computation of this function would provide new relevant CFT data: apart from the corrections to the canonical conformal dimensions (5.20), it also contains, in the corresponding OPE limits, all the super-conformal properties of the next members of the family of the non-BPS twisted Ramond fields.

Relevant information about fuzzball microstates can be extracted from four-point functions similar to (1.2), but with the deformation operators replaced by NS chiral fields $O_{[2]}^{(p, p)}$, with dimensions $\Delta_{2}^{(0,0)}=1$ and $\Delta_{2}^{(1,1)}=2$, viz.

$$
\left\langle R_{[n]}^{-}(\infty) O_{[2]}^{(p, p)}(1) O_{[2]}^{(p, p)}(u, \bar{u}) R_{[m]}^{+}(0)\right\rangle .
$$

Computing these functions by the methods of section 4 is actually easier than computing (1.2). Their short-distance limits contain the CFT data - the conformal dimensions and structure constants - about the non-BPS fields $X_{n \pm 1}^{(p, p) \pm}$ appearing in the OPEs $O_{[2]}^{(p, p)} R_{n}^{ \pm}$.

R-neutral Ramond ground states. Here we have focused on the R-charged $\mathrm{SU}(2)_{L, R}$ doublets $R_{[n]}^{\alpha}$. The remaining Ramond fields, that are neutral under R-symmetry and form a doublet of $\mathrm{SU}(2)_{2}$, have only been mentioned in passing. The renormalization of such fields with a single cycle, $R_{[n]}^{\dot{A}}$, can be studied with the same methods of the present paper. One must compute their four-point function with $O_{[2]}^{(\mathrm{int})}$ by lifting to covering space with the corresponding R-neutral spin fields $S^{\dot{A}}$, etc. In practice, the actual computation of the four-point function is more complicated then the one presented in section 4.2 , because some simplifying cancelations only occur for the R-charged fields. Once the function is found, however, all the methodology developed here for exploration of operator algebras via short-distance limits, as well as the renormalization scheme, can be applied. We have presented these results elsewhere [72].

Lifted vs. protected states. Supergravity solutions of fuzzballs correspond to states in the CFT where all component strings are in a Ramond ground state, i.e. $\prod_{k}\left(R_{\left[n_{k}\right]}^{\alpha_{k}}\right)^{k}$, with $\sum_{k} k n_{k}=N$. If there is only one $n$-wound string, then this state becomes $\left(R_{[1]}^{\beta}\right)^{N-n} R_{[n]}^{\alpha}$, where the untwisted Ramond field $R_{[1]}^{\beta}$ is a (symmetrized) spin field. The fields that we have found to be renormalized are made by putting the $n$-wound string is in a Ramond ground state, while all the other untwisted strings are in the NS vacuum, i.e. explicitly $R_{[n]}^{\alpha} \cong(\mathbb{1})^{N-n} R_{[n]}^{\alpha}$. The fact that one can define a spectral flow of the $c=6 n$ superconformal algebra in the $n$-wound string at the free orbifold point may suggest that both $(\mathbb{1})^{N-n} R_{[n]}^{\alpha}$ and $\left(R_{[1]}^{\beta}\right)^{N-n} R_{[n]}^{\alpha}$ are protected. As we have shown here, this is not true.

The single-cycle Ramond field with maximal twist $n=N$, which is a "pure" Ramond ground state in the full orbifold theory, is, indeed, protected, as far as our analysis goes: the four-point function for this field scales as $1 / N^{2}$ and must be computed with a genusone covering surface. The interesting feature that our calculation highlights is that this protection depends crucially on the combinatorics involved in the combinations of the permutation cycles of the twisted fields $R_{[n]}^{\alpha}$ and $O_{[2]}^{(\mathrm{int})}$. The protection is due not to the 
fact that the four-point function or its integral vanish - in contrast to what happens in the case of minimal twist $n=2$, where the behavior of the four-point functions changes drastically and $J_{R}(2)$ vanishes, neither the function $z(t)$, the map $u(x)$, the functions $G_{R}(x)$, nor the integrals $J_{R}(n)$, none of them is able to distinguish between $2<n<N$ or $n=N$. What separates the maximal twist is the analysis of eq. (4.18), which dictates the $N$-dependence of $G_{R}(u, \bar{u})$, and implies that any function obtained from the genus-zero covering map must correspond to a permutation of $S_{N}$ such that $n<N$. These features become starker when we consider the composite field $\left(R_{\left[n_{1}\right]}^{\alpha_{1}} R_{\left[n_{2}\right]}^{\alpha_{2}}\right) \cong(\mathbb{1})^{N-n_{1}-n_{2}} R_{\left[n_{1}\right]}^{\alpha_{1}} R_{\left[n_{2}\right]}^{\alpha_{2}}$, which was done in [59]. When $n_{1}+n_{2}<N$, the solutions to the equation equivalent to eq. (4.18) allow for factorizations of

$$
\left\langle\left(R_{\left[n_{1}\right]}^{\alpha_{1}} R_{\left[n_{2}\right]}^{\alpha_{2}}\right]^{\dagger}(\infty, \bar{\infty}) O_{[2]}^{(\mathrm{int})}(1, \overline{1}) O_{[2]}^{(\mathrm{int})}(u, \bar{u})\left(R_{\left[n_{1}\right]}^{\alpha_{1}} R_{\left[n_{2}\right]}^{\alpha_{2}}\right)(0, \overline{0})\right\rangle
$$

into four-point functions involving only one of the single-cycle fields; then the composite field renormalizes as a corollary of our present results. When $n_{1}+n_{2}=N$, and the field becomes a "pure" Ramond ground state with $h=\frac{1}{24} c_{\text {orb }}$, there is no factorization. Now, the covering surface for this completely connected function has genus zero, and we can calculate the four-point function and its integral explicitly, in contrast to what happened here for the field $R_{[N]}^{\alpha}$. The four-point function we find in [59] is non-trivial, and reveals conformal data and OPE fusion rules. Its integral, corresponding to $J_{R}$, is also non-trivial but it does vanish after we apply our regularization procedure and the DF construction: thus we see explicitly that the family of pure Ramond fields $\left(R_{\left[n_{1}\right]}^{\alpha_{1}} R_{\left[n_{2}\right]}^{\alpha_{2}}\right)$ with $n_{1}+n_{2}=N$ is again protected.

In closing, one cannot avoid the question of what are (if any) the bulk holographic images of the renormalized $R_{[n]}^{\alpha}$ fields, with their continuous, $\lambda$-dependent conformal dimensions. The answer remains to be discovered, and there are indications that tools necessary for this end include the description of the symmetry algebra of the deformed $\mathrm{SCFT}_{2}$ and its unitary representations.

\section{Acknowledgments}

The work of M.S. is partially supported by the Bulgarian NSF grant KP-06-H28/5 and that of M.S. and G.S. by the Bulgarian NSF grant KP-06-H38/11. M.S. is grateful for the kind hospitality of the Federal University of Espírito Santo, Vitória, Brazil, where part of his work was done.

\section{A Conventions for the $\mathcal{N}=(4,4)$ SCFT}

In the $\mathcal{N}=(4,4)$ superalgebra, the R-currents $J^{a}(z), \tilde{J}^{a}(\bar{z})$, and the supercurrents $G^{\alpha A}(z)$, $\tilde{G}^{\dot{\alpha} \dot{A}}(\bar{z})$ have indices in SU(2) groups as follows: $a=1,2,3$ and $\dot{a}=\dot{1}, \dot{2}, \dot{3}$ transform as a triplets of $\mathrm{SU}(2)_{L}$ and $\mathrm{SU}(2)_{R}$, respectively; $\alpha=+,-$ and $\dot{\alpha}=\dot{+}, \dot{-}$ transform as a doublets of $\mathrm{SU}(2)_{L}$ and $\mathrm{SU}(2)_{R}$, respectively; indices $A=1,2$ and $\dot{A}=\dot{1}, \dot{2}$ transform as doublets of $\mathrm{SU}(2)_{1}$ and $\mathrm{SU}(2)_{2}$, respectively. 
The SCFT can be realized in terms of four real bosons $X_{i}(z, \bar{z})$, four real holomorphic fermions $\psi_{i}(z)$ and four real anti-holomorphic fermions $\tilde{\psi}_{i}(\bar{z})$, with $i=1, \cdots, 4$. They are related to the complex fields $X^{\dot{A} A}(z, \bar{z}), \psi^{\alpha \dot{A}}(z)$ and $\tilde{\psi}^{\dot{\alpha} \dot{A}}(\bar{z})$ by

$$
\begin{aligned}
X_{\dot{A} A} & =\frac{1}{\sqrt{ } 2} X_{i}\left[\sigma^{i}\right]_{\dot{A} A}=\frac{1}{\sqrt{ } 2}\left[\begin{array}{cc}
X_{3}+i X_{4} & X_{1}-i X_{2} \\
X_{1}+i X_{2} & -X_{3}+i X_{4}
\end{array}\right], \\
\psi^{\alpha \dot{1}} & =\left[\begin{array}{l}
\psi^{+1} \\
\psi^{-1}
\end{array}\right]=\frac{1}{\sqrt{ } 2}\left[\begin{array}{l}
\psi_{1}+i \psi_{2} \\
\psi_{3}+i \psi_{4}
\end{array}\right], \quad \psi^{\alpha \dot{2}}=\left[\begin{array}{c}
\psi^{+\dot{2}} \\
\psi^{-\dot{2}}
\end{array}\right]=\frac{1}{\sqrt{ } 2}\left[\begin{array}{c}
\psi_{3}-i \psi_{4} \\
-\psi_{1}+i \psi_{2}
\end{array}\right] .
\end{aligned}
$$

There are analogous constructions for the right-moving sector. The Levi-Civita symbol always has the structure $\epsilon^{12}=+1$. Pauli matrices are defined such that $\sigma^{3}=\operatorname{Diag}(1,-1)$. The "Pauli vector" $\sigma^{i}=\left(\sigma^{1}, \sigma^{2}, \sigma^{3}, \sigma^{4}\right)$ and its conjugate $\bar{\sigma}^{i}$ have components (we work in Euclidean space) $\sigma^{a}=-\bar{\sigma}^{a} \sigma^{4}=i \mathbb{1}_{2 \times 2}=\bar{\sigma}^{4}$.

The reality condition of $X_{i}$ and $\psi_{i}$ implies that

$$
X^{\dot{A} A} \equiv-\epsilon^{\dot{A} \dot{B}} \epsilon^{A B} X_{\dot{B} B}, \quad\left(X_{\dot{A} A}\right)^{\dagger}=X^{\dot{A} A}, \quad\left(\psi^{\alpha \dot{A}}\right)^{\dagger}=\psi_{\alpha \dot{A}}
$$

Two-point functions are

$$
\begin{aligned}
\left\langle\partial X^{\dot{A} A}(z) \partial X^{\dot{B} B}\left(z^{\prime}\right)\right\rangle & =\frac{2 \epsilon^{\dot{A} \dot{B}} \epsilon^{A B}}{\left(z-z^{\prime}\right)^{2}} \\
\left\langle\psi^{\alpha \dot{A}}(z) \psi^{\beta \dot{B}}\left(z^{\prime}\right)\right\rangle & =-\frac{\epsilon^{\alpha \beta} \epsilon^{\dot{A} \dot{B}}}{z-z^{\prime}} \\
\left\langle\partial \phi_{r}(z) \partial \phi_{s}\left(z^{\prime}\right)\right\rangle & =-\frac{\delta_{r s}}{\left(z-z^{\prime}\right)^{2}}
\end{aligned}
$$

where the last equation is for the bosonized fermions (2.2). The non-vanishing bosonic two-point functions are between a current $\partial X^{\dot{A} A}$ and its complex conjugate; explicitly,

$$
\left\langle\partial X^{\mathrm{i} 1}(z)\left(\partial X^{\mathrm{i} 1}\right)^{\dagger}\left(z^{\prime}\right)\right\rangle=\frac{2}{\left(z-z^{\prime}\right)^{2}}, \quad\left\langle\partial X^{\mathrm{i} 2}(z)\left(\partial X^{\mathrm{i} 2}\right)^{\dagger}\left(z^{\prime}\right)\right\rangle=-\frac{2}{\left(z-z^{\prime}\right)^{2}},
$$

as can be checked from (A.4) using the reality conditions (A.3).

\section{B Asymptotics to OPEs}

In calculating OPEs, we need to know the inverse of (4.6) near the base-sphere points $u_{*}=0, \infty, 1$. When $u_{*}=0$, the roots of eq. (4.17) are obvious: $x=0$ (with multiplicity $n-1$ ) and $x=-n$ (with multiplicity $n+1$ ). Going back to (4.6), we find the form of $u(x)$ in these two limits,

$$
u(x) \approx \frac{n^{n+1}}{(1-n)^{n-1}} x^{n-1}, \quad u(x) \approx \frac{n^{n-1}}{(-n-1)^{n+1}}(x+n)^{n+1}
$$

so inverting we get the two functions

$$
(u \rightarrow 0) \quad\left[\begin{array}{ll}
x \rightarrow 0, & x_{1}^{0}(u) \approx\left(\frac{(1-n)^{n-1}}{n^{n+1}} u\right)^{\frac{1}{n-1}} \\
x \rightarrow-n, & x_{2}^{0}(u) \approx-n+\left(\frac{(-n-1)^{n+1}}{n^{n-1}} u\right)^{\frac{1}{n+1}}
\end{array}\right.
$$


Taking $u_{*}=\infty$, eq. (4.17) reduces to $(x-1)^{n+1}(x+n-1)^{n-1}=0$, with roots $x=1$ and $x=1-n$. The function $u(x)$ behaves in these limits as

$$
u(x) \approx \frac{(1+n)^{n+1}}{n^{n-1}} \frac{1}{(x-1)^{n+1}}, \quad u(x) \approx \frac{(n-1)^{n-1}}{n^{n+1}} \frac{1}{(x-1+n)^{n-1}}
$$

so we have the inverse functions

$$
(u \rightarrow \infty)\left[\begin{array}{ll}
x \rightarrow 1-n, & x_{1}^{\infty}(u) \approx 1-n+\left(\frac{(n-1)^{n-1}}{n^{n+1}} \frac{1}{u}\right)^{\frac{1}{n-1}} \\
x \rightarrow 1, & x_{2}^{\infty}(u) \approx 1+\left(\frac{(1+n)^{n+1}}{n^{n-1}} \frac{1}{u}\right)^{\frac{1}{n+1}}
\end{array}\right.
$$

When $u_{*}=1$, one cannot find the $2 n$ solutions of eq. (4.17), but fortunately we are only interested in those solutions which also correspond to the limit $t_{1} \rightarrow x$. In this case, instead of a polynomial equation of degree $2 n$, we must solve eq. (4.5) which becomes

$$
\frac{2 x+n-1}{(n+x) x}=0
$$

with only two solutions: $x=\infty$ and $x=\frac{1}{2}(1-n)$. The behavior of $u(x)$ near $x=\infty$ can be found with the conformal transformation $x=1 / \varepsilon$; evaluating $u(1 / \varepsilon)$ around small $\varepsilon$,

$$
u(1 / \varepsilon)=1+4 n \varepsilon+2 n(1+3 n) \varepsilon^{2}+\mathrm{O}\left(\varepsilon^{3}\right)
$$

while expanding $u(x)$ the second limit, when $x \rightarrow \frac{1-n}{2}$, we get

$$
\begin{aligned}
u(x)= & 1-\frac{64 n}{3\left(n^{2}-1\right)^{2}}\left(x-\frac{1-n}{2}\right)^{3}-\frac{512 n\left(1+n^{2}\right)}{5\left(n^{2}-1\right)^{4}}\left(x-\frac{1-n}{2}\right)^{5} \\
& +\frac{2048 n^{2}}{9\left(n^{2}-1\right)^{4}}\left(x-\frac{1-n}{2}\right)^{6}+\mathrm{O}\left(x-\frac{1-n}{2}\right)^{7}
\end{aligned}
$$

Inverting the two series above, we find

$$
(u \rightarrow 1) \quad\left[\begin{array}{rl}
x \rightarrow \infty, \quad x_{1}^{1}(u) \approx & -\frac{4 n}{1-u}+\frac{3 n+1}{2}+\cdots \\
x \rightarrow \frac{1-n}{2}, \quad x_{2}^{1}(u) \approx & \frac{1-n}{2} \\
& +\frac{3^{1 / 3}\left(n^{2}-1\right)^{2 / 3}}{4 n^{1 / 3}}(1-u)^{\frac{1}{3}} \\
& -\frac{3\left(n^{2}+1\right)}{40 n}(1-u) \\
& +\frac{\left(n^{2}-1\right)^{2 / 3}}{8 \cdot 3^{2 / 3} \cdot n^{1 / 3}}(1-u)^{\frac{4}{3}}+\cdots
\end{array}\right.
$$

Note that the multiplicity of the solution $x=\frac{1-n}{2}$ is 3 , and that of $x=\infty$ is 1 .

\section{OPEs with bare twists and structure constants}

In this appendix, we examine the OPE limits of the functions $G_{\sigma}(x)$ and $g(x)$. We derive several structure constants, some of which are known in the literature, thus checking our expressions for $g(x)$ and $G_{\sigma}(x)$. 
We start with the limit $u \rightarrow 1$ for $G(u, \bar{u})$. The identity channel is the same as for the Ramond fields discussed in the text, while the second channel gives

$$
G_{\sigma}\left(x_{2}^{1}(u)\right)=\frac{d_{4}}{(1-u)^{4 / 3}}+\frac{d_{2}}{(1-u)^{2 / 3}}+\frac{d_{1}}{(1-u)^{1 / 3}}+\text { non-sing. }
$$

where, after taking (5.6) into account,

$$
\begin{aligned}
\log \left|d_{4}\right|^{2}= & \left(n+\frac{1}{n}+\frac{2}{3}\right) \log (n+1)-\left(n+\frac{1}{n}-\frac{2}{3}\right) \log (n-1)-\frac{4}{3} \log n \\
& -4 \log 2-\frac{8}{3} \log 3 .
\end{aligned}
$$

The powers of $u$ reveal the conformal family of $\sigma_{3}$ with no operator of dimension one among the descendants. Inserting the OPE (5.8) back into the four-point function, we find that

$$
\left|d_{4}\right|^{2}=\left\langle O_{2}^{(\mathrm{int})}(\infty) \sigma_{3}(1) O_{2}^{(\mathrm{int})}(0)\right\rangle\left\langle\sigma_{n}(\infty) \sigma_{3}(1) \sigma_{n}(0)\right\rangle .
$$

The structure constant $C_{232}^{O^{(\text {int })} \sigma O^{(\text {int })}}=\left\langle O_{2}^{(\text {int })}(\infty) \sigma_{3}(1) O_{2}^{(\text {int })}(0)\right\rangle$ carries no $n$-dependence, and we can write $C_{n 3 n}^{\sigma}=\left\langle\sigma_{n}(\infty) \sigma_{3}(1) \sigma_{n}(0)\right\rangle$ as

$$
\log C_{n 3 n}^{\sigma}=\left(n+\frac{1}{n}+\frac{2}{3}\right) \log (n+1)-\left(n+\frac{1}{n}-\frac{2}{3}\right) \log (n-1)-\frac{4}{3} \log n+\kappa,
$$

which agrees with eq. (6.25) of ref. [12], apart from an overall factor of $\frac{1}{6}$. The $n$-independent number $\kappa=\frac{4}{3} \log 3+\frac{1}{3} \log 2$ can be obtained by taking $n=2$ in eq. (C.4), and comparing with eq. (C.10), below. Hence

$$
\log C_{232}^{O^{(\mathrm{int})} \sigma O^{(\mathrm{int})}}=4 \log 3+\frac{13}{3} \log 2 .
$$

An interesting check of the results above comes from the function (4.16), whose limit $u \rightarrow 1$ now gives the $\mathrm{OPE} \sigma_{2}(u) \sigma_{2}(1)$. Counting powers of $u$ in

$$
\begin{aligned}
& g\left(x_{1}^{1}(u)\right)=\frac{c_{\sigma}(-4 n)^{3 / 4}}{(1-u)^{3 / 4}}+\mathrm{O}(1-u)^{1 / 4} \\
& g\left(x_{2}^{1}(u)\right)=\frac{b}{(1-u)^{\frac{1}{12}}}+\mathrm{O}(1-u)^{7 / 12}
\end{aligned}
$$

Eq. (C.6) gives again the identity, thus determining $c_{\sigma}=(-4 n)^{-3 / 4}$. In the channel (C.7) we find $\sigma_{3}$ with its dimension $h_{3}^{\sigma}=\frac{2}{3}$, completing the well-known fusion rule $\left[\sigma_{2}\right] \times\left[\sigma_{2}\right]=$ $\left[\sigma_{1}\right]+\left[\sigma_{3}\right]$. Note the absence of next-to leading singularities in eqs. (C.6) and (C.7); there are no descendants in these OPEs.

The constant $b$ gives information about $\left\langle\sigma_{p} \sigma_{q} \sigma_{r}\right\rangle=C_{p q r}^{\sigma}$ :

$$
\begin{aligned}
\log C_{n 3 n}^{\sigma}+\log C_{232}^{\sigma}= & \log |b|^{2} \\
= & \left(n+\frac{1}{n}+\frac{2}{3}\right) \log (n+1)-\left(n+\frac{1}{n}-\frac{2}{3}\right) \log (n-1) \\
& -\frac{4}{3} \log n-4 \log 2-\frac{1}{6} \log 3
\end{aligned}
$$


Comparison with eqs. (C.3) and (C.2) reveals the same $n$-dependence for both three-point functions - an important cross-check between the two $g(x)$ and $G_{\sigma}(x)$ (which were obtained independently).

The more general fusion rule

$$
\left[\sigma_{2}\right] \times\left[\sigma_{n}\right]=\left[\sigma_{n-1}\right]+\left[\sigma_{n+1}\right]
$$

can be derived from

$$
g\left(x_{1}^{0}(u)\right)=u^{-\frac{5 n^{2}-5 n+2}{8 n(n-1)}}\left(c_{1}^{-}+c_{2}^{-} u^{\frac{1}{n-1}}+\cdots\right), \quad g\left(x_{2}^{0}(u)\right)=u^{-\frac{5 n^{2}+5 n-2}{8 n(n+1)}}\left(c_{1}^{+}+c_{2}^{+} u^{\frac{1}{n+1}}+\cdots\right)
$$

where the coefficients $c_{1}^{ \pm}$are readily computable. One can check from the powers of $u$ that channels $x_{1}^{0}(u)$ and $x_{2}^{0}(u)$ give operators of dimensions $h_{n-1}^{\sigma}$ and $h_{n+1}^{\sigma}$, that is $\sigma_{n-1}$ and $\sigma_{n+1}$, respectively. The coefficients $c_{1}^{ \pm}$give us information about $\left\langle\sigma_{n}(\infty) \sigma_{2}(1) \sigma_{n-1}(0)\right\rangle=$ $\left|c_{1}^{-}\right|$and $\left\langle\sigma_{n}(\infty) \sigma_{2}(1) \sigma_{n+1}(0)\right\rangle=\left|c_{1}^{+}\right|$. Explicitly

$$
\begin{aligned}
& \log C_{n, 2, n-1}^{\sigma}=-\frac{2 n^{2}-n+2}{4 n} \log (n-1)+\frac{2 n^{2}-3 n+3}{4(n-1)} \log n-\frac{5}{4} \log 2 \\
& \log C_{n, 2, n+1}^{\sigma}=+\frac{2 n^{2}+n+2}{4 n} \log (n+1)-\frac{2 n^{2}+3 n+3}{4 n(n+1)} \log n-\frac{5}{4} \log 2
\end{aligned}
$$

This agrees with the result of ref. [12], again, apart from an overall factor of $\frac{1}{6}$. Inserting $n=3$ in eq. (C.10), we find that $\log C_{3,2,2}^{\sigma}=-\frac{5}{3} \log 2$, which was used to derived eq. (C.5).

Open Access. This article is distributed under the terms of the Creative Commons Attribution License (CC-BY 4.0), which permits any use, distribution and reproduction in any medium, provided the original author(s) and source are credited.

\section{References}

[1] J.M. Maldacena, The large $N$ limit of superconformal field theories and supergravity, Int. $J$. Theor. Phys. 38 (1999) 1113 [Adv. Theor. Math. Phys. 2 (1998) 231] [hep-th/9711200] [INSPIRE].

[2] J.M. Maldacena and A. Strominger, $A d S_{3}$ black holes and a stringy exclusion principle, JHEP 12 (1998) 005 [hep-th/9804085] [INSPIRE].

[3] N. Seiberg and E. Witten, The D1/D5 system and singular CFT, JHEP 04 (1999) 017 [hep-th/9903224] [INSPIRE].

[4] J.R. David, G. Mandal and S.R. Wadia, Microscopic formulation of black holes in string theory, Phys. Rept. 369 (2002) 549 [hep-th/0203048] [INSPIRE].

[5] S.D. Mathur, The fuzzball proposal for black holes: An elementary review, Fortsch. Phys. 53 (2005) 793 [hep-th/0502050] [INSPIRE].

[6] K. Skenderis and M. Taylor, The fuzzball proposal for black holes, Phys. Rept. 467 (2008) 117 [arXiv: 0804.0552] [INSPIRE].

[7] A. Strominger and C. Vafa, Microscopic origin of the Bekenstein-Hawking entropy, Phys. Lett. B 379 (1996) 99 [hep-th/9601029] [INSPIRE]. 
[8] O. Lunin and S.D. Mathur, AdS/CFT duality and the black hole information paradox, Nucl. Phys. B 623 (2002) 342 [hep-th/0109154] [inSPIRE].

[9] I. Kanitscheider, K. Skenderis and M. Taylor, Fuzzballs with internal excitations, JHEP 06 (2007) 056 [arXiv: 0704.0690] [INSPIRE].

[10] I. Kanitscheider, K. Skenderis and M. Taylor, Holographic anatomy of fuzzballs, JHEP 04 (2007) 023 [hep-th/0611171] [INSPIRE].

[11] S.D. Mathur and D. Turton, The fuzzball nature of two-charge black hole microstates, Nucl. Phys. B 945 (2019) 114684 [arXiv:1811.09647] [INSPIRE].

[12] O. Lunin and S.D. Mathur, Correlation functions for MN/SN orbifolds, Commun. Math. Phys. 219 (2001) 399 [hep-th/0006196] [INSPIRE].

[13] O. Lunin and S.D. Mathur, Three point functions for $M^{N} / S_{N}$ orbifolds with $\mathcal{N}=4$ supersymmetry, Commun. Math. Phys. 227 (2002) 385 [hep-th/0103169] [INSPIRE].

[14] V. Balasubramanian, P. Kraus and M. Shigemori, Massless black holes and black rings as effective geometries of the D1-D5 system, Class. Quant. Grav. 22 (2005) 4803 [hep-th/0508110] [INSPIRE].

[15] S.G. Avery, B.D. Chowdhury and S.D. Mathur, Emission from the D1D5 CFT, JHEP 10 (2009) 065 [arXiv:0906.2015] [inSPIRE].

[16] A. Pakman, L. Rastelli and S.S. Razamat, Extremal Correlators and Hurwitz Numbers in Symmetric Product Orbifolds, Phys. Rev. D 80 (2009) 086009 [arXiv:0905.3451] [INSPIRE].

[17] A. Pakman, L. Rastelli and S.S. Razamat, A Spin Chain for the Symmetric Product CFT(2), JHEP 05 (2010) 099 [arXiv:0912.0959] [INSPIRE].

[18] B.A. Burrington, A.W. Peet and I.G. Zadeh, Operator mixing for string states in the D1-D5 CFT near the orbifold point, Phys. Rev. D 87 (2013) 106001 [arXiv:1211.6699] [INSPIRE].

[19] I. Bena and N.P. Warner, Resolving the Structure of Black Holes: Philosophizing with a Hammer, arXiv:1311.4538 [INSPIRE].

[20] Z. Carson, S. Hampton, S.D. Mathur and D. Turton, Effect of the deformation operator in the D1D5 CFT, JHEP 01 (2015) 071 [arXiv:1410.4543] [INSPIRE].

[21] Z. Carson, S. Hampton and S.D. Mathur, Second order effect of twist deformations in the D1D5 CFT, JHEP 04 (2016) 115 [arXiv:1511.04046] [INSPIRE].

[22] A.L. Fitzpatrick, J. Kaplan, D. Li and J. Wang, On information loss in $A d S_{3} / C F T_{2}, J H E P$ 05 (2016) 109 [arXiv: 1603.08925] [INSPIRE].

[23] B.A. Burrington, I.T. Jardine and A.W. Peet, Operator mixing in deformed D1D5 CFT and the OPE on the cover, JHEP 06 (2017) 149 [arXiv:1703.04744] [INSPIRE].

[24] A. Galliani, S. Giusto and R. Russo, Holographic 4-point correlators with heavy states, JHEP 10 (2017) 040 [arXiv: 1705.09250] [INSPIRE].

[25] A. Bombini, A. Galliani, S. Giusto, E. Moscato and R. Russo, Unitary 4-point correlators from classical geometries, Eur. Phys. J. C 78 (2018) 8 [arXiv:1710.06820] [INSPIRE].

[26] J. Garcia i Tormo and M. Taylor, Correlation functions in the D1-D5 orbifold CFT, JHEP 06 (2018) 012 [arXiv: 1804.10205] [inSPIRE].

[27] L. Eberhardt, M.R. Gaberdiel and R. Gopakumar, The Worldsheet Dual of the Symmetric Product CFT, JHEP 04 (2019) 103 [arXiv:1812.01007] [INSPIRE]. 
[28] I. Bena, P. Heidmann, R. Monten and N.P. Warner, Thermal Decay without Information Loss in Horizonless Microstate Geometries, SciPost Phys. 7 (2019) 063 [arXiv: 1905.05194] [INSPIRE].

[29] A. Dei, L. Eberhardt and M.R. Gaberdiel, Three-point functions in $A d S_{3} / C F T_{2}$ holography, JHEP 12 (2019) 012 [arXiv:1907.13144] [INSPIRE].

[30] L. Eberhardt, M.R. Gaberdiel and R. Gopakumar, Deriving the $A d S_{3} / C F T_{2}$ correspondence, JHEP 02 (2020) 136 [arXiv:1911.00378] [INSPIRE].

[31] S. Giusto, R. Russo and C. Wen, Holographic correlators in AdS 3 , JHEP 03 (2019) 096 [arXiv: 1812.06479] [INSPIRE].

[32] E.J. Martinec, S. Massai and D. Turton, Little Strings, Long Strings, and Fuzzballs, JHEP 11 (2019) 019 [arXiv: 1906.11473] [INSPIRE].

[33] S. Hampton and S.D. Mathur, Thermalization in the D1D5 CFT, JHEP 06 (2020) 004 [arXiv: 1910.01690] [INSPIRE].

[34] N.P. Warner, Lectures on Microstate Geometries, arXiv:1912.13108 [INSPIRE].

[35] A. Dei and L. Eberhardt, Correlators of the symmetric product orbifold, JHEP 01 (2020) 108 [arXiv: 1911.08485] [INSPIRE].

[36] A. Galliani, S. Giusto, E. Moscato and R. Russo, Correlators at large c without information loss, JHEP 09 (2016) 065 [arXiv: 1606.01119] [INSPIRE].

[37] A. Bombini and A. Galliani, AdS $S_{3}$ four-point functions from $\frac{1}{8}$-BPS states, JHEP 06 (2019) 044 [arXiv: 1904.02656] [INSPIRE].

[38] S. Giusto, M.R.R. Hughes and R. Russo, The Regge limit of $A d S_{3}$ holographic correlators, JHEP 11 (2020) 018 [arXiv:2007.12118] [INSPIRE].

[39] B. Guo and S.D. Mathur, Lifting of states in 2-dimensional $N=4$ supersymmetric CFTs, JHEP 10 (2019) 155 [arXiv:1905.11923] [INSPIRE].

[40] C.A. Keller and I.G. Zadeh, Lifting $\frac{1}{4}$-BPS States on K3 and Mathieu Moonshine, Commun. Math. Phys. 377 (2020) 225 [arXiv:1905.00035] [INSPIRE].

[41] C.A. Keller and I.G. Zadeh, Conformal Perturbation Theory for Twisted Fields, J. Phys. A 53 (2020) 095401 [arXiv: 1907.08207] [InSPIRE].

[42] B. Guo and S.D. Mathur, Lifting of level-1 states in the D1D5 CFT, JHEP 03 (2020) 028 [arXiv: 1912.05567] [INSPIRE].

[43] A. Belin, A. Castro, C.A. Keller and B. Mühlmann, The Holographic Landscape of Symmetric Product Orbifolds, JHEP 01 (2020) 111 [arXiv:1910.05342] [INSPIRE].

[44] B. Guo and S.D. Mathur, Lifting at higher levels in the D1D5 CFT, JHEP 11 (2020) 145 [arXiv: 2008.01274] [INSPIRE].

[45] S.G. Avery, B.D. Chowdhury and S.D. Mathur, Deforming the D1D5 CFT away from the orbifold point, JHEP 06 (2010) 031 [arXiv:1002.3132] [INSPIRE].

[46] S.G. Avery, B.D. Chowdhury and S.D. Mathur, Excitations in the deformed D1D5 CFT, JHEP 06 (2010) 032 [arXiv: 1003.2746] [INSPIRE].

[47] Z. Carson, S. Hampton and S.D. Mathur, Full action of two deformation operators in the D1D5 CFT, JHEP 11 (2017) 096 [arXiv:1612.03886] [INSPIRE]. 
[48] A.A. Lima, G.M. Sotkov and M. Stanishkov, Microstate Renormalization in Deformed D1-D5 SCFT, Phys. Lett. B 808 (2020) 135630 [arXiv:2005.06702] [InSPIRE].

[49] L.J. Dixon, J.A. Harvey, C. Vafa and E. Witten, Strings on Orbifolds, Nucl. Phys. B 261 (1985) 678 [INSPIRE].

[50] G.E. Arutyunov and S.A. Frolov, Four graviton scattering amplitude from $S^{* *} N R^{8}$ supersymmetric orbifold sigma model, Nucl. Phys. B 524 (1998) 159 [hep-th/9712061] [INSPIRE].

[51] G.E. Arutyunov and S.A. Frolov, Virasoro amplitude from the $S^{N} R^{24}$ orbifold sigma model, Theor. Math. Phys. 114 (1998) 43 [hep-th/9708129] [inSPIRE].

[52] A. Pakman, L. Rastelli and S.S. Razamat, Diagrams for Symmetric Product Orbifolds, JHEP 10 (2009) 034 [arXiv: 0905. 3448] [inSPIRE].

[53] V.S. Dotsenko and V.A. Fateev, Conformal Algebra and Multipoint Correlation Functions in Two-Dimensional Statistical Models, Nucl. Phys. B 240 (1984) 312 [InSPIRE].

[54] V.S. Dotsenko and V.A. Fateev, Four Point Correlation Functions and the Operator Algebra in the Two-Dimensional Conformal Invariant Theories with the Central Charge $c<1$, Nucl. Phys. B 251 (1985) 691 [inSPIRE].

[55] V.S. Dotsenko, Lectures on conformal field theory, in Conformal Field Theory and Solvable Lattice Models, pp. 123-170, Mathematical Society of Japan, (1988).

[56] G. Mussardo, G. Sotkov and M. Stanishkov, Ramond Sector of the Supersymmetric Minimal Models, Phys. Lett. B 195 (1987) 397 [InSPIRE].

[57] G. Mussardo, G. Sotkov and H. Stanishkov, Fine Structure of the Supersymmetric Operator Product Expansion Algebras, Nucl. Phys. B 305 (1988) 69 [INSPIRE].

[58] G. Mussardo, G. Sotkov and M. Stanishkov, $N=2$ superconformal minimal models, Int. J. Mod. Phys. A 4 (1989) 1135 [inSPIRE].

[59] A.A. Lima, G.M. Sotkov and M. Stanishkov, Correlation functions of composite Ramond fields in deformed D1-D5 orbifold SCFT 2 , Phys. Rev. D 102 (2020) 106004 [arXiv: 2006.16303] [INSPIRE].

[60] B.A. Burrington, I.T. Jardine and A.W. Peet, The OPE of bare twist operators in bosonic $S_{N}$ orbifold CFTs at large N, JHEP 08 (2018) 202 [arXiv: 1804.01562] [INSPIRE].

[61] T. De Beer, B.A. Burrington, I.T. Jardine and A.W. Peet, The large $N$ limit of OPEs in symmetric orbifold CFTs with $\mathcal{N}=(4,4)$ supersymmetry, JHEP 08 (2019) 015 [arXiv: 1904.07816] [INSPIRE].

[62] L.J. Dixon, D. Friedan, E.J. Martinec and S.H. Shenker, The Conformal Field Theory of Orbifolds, Nucl. Phys. B 282 (1987) 13 [INSPIRE].

[63] R. Dijkgraaf, G.W. Moore, E.P. Verlinde and H.L. Verlinde, Elliptic genera of symmetric products and second quantized strings, Commun. Math. Phys. 185 (1997) 197 [hep-th/9608096] [INSPIRE].

[64] K. Roumpedakis, Comments on the $S_{N}$ orbifold CFT in the large N-limit, JHEP 07 (2018) 038 [arXiv: 1804.03207] [INSPIRE].

[65] S.K. Lando and A.K. Zvonkin, Graphs on surfaces and their applications, vol. 141. Springer Science \& Business Media, (2013). 
[66] F.W.J. Olver et al. eds., NIST Digital Library of Mathematical Functions, release 1.0.24 of 2019-09-15, http://dlmf.nist.gov/.

[67] H. Bateman, Higher Transcendental Functions, vol. I-III. McGraw-Hill Book Company, (1953).

[68] A. Jevicki, M. Mihailescu and S. Ramgoolam, Gravity from $C F T$ on $S^{* *} N(X)$ : Symmetries and interactions, Nucl. Phys. B 577 (2000) 47 [hep-th/9907144] [INSPIRE].

[69] A. Schwimmer and N. Seiberg, Comments on the $N=2, N=3, N=4$ Superconformal Algebras in Two-Dimensions, Phys. Lett. B 184 (1987) 191 [INSPIRE].

[70] A. Sen, On the Background Independence of String Field Theory, Nucl. Phys. B 345 (1990) 551 [INSPIRE].

[71] M. Campbell, P.C. Nelson and E. Wong, Stress tensor perturbations in conformal field theory, Int. J. Mod. Phys. A 6 (1991) 4909 [inSPIRE].

[72] A.A. Lima, G.M. Sotkov and M. Stanishkov, Dynamics of R-neutral Ramond fields in the D1-D5 SCFT, arXiv:2012.08021 [INSPIRE]. 